\title{
A triple-negative breast cancer surrogate subtype classification that correlates with gene expression subtypes
}

\section{Tae-Kyung Yoo}

Catholic University of Korea College of Medicine: Catholic University of Korea School of Medicine https://orcid.org/0000-0002-5790-353X

\section{Jun Kang}

Catholic University of Korea College of Medicine: Catholic University of Korea School of Medicine

\section{Awon Lee}

Catholic University of Korea College of Medicine: Catholic University of Korea School of Medicine Byung Joo Chae ( $\sim$ bjchae@gmail.com )

Samsung Medical Center, Sungkyunkwan University https://orcid.org/0000-0003-1564-0978

\section{Research article}

Keywords: Triple Negative Breast Neoplasms, Classification, Biomarkers, Receptors, Androgen, Lymphocytes, Tumor-Infiltration, Cyclin-Dependent Kinase Inhibitor p16

Posted Date: July 13th, 2021

DOI: https://doi.org/10.21203/rs.3.rs-698148/v1

License: (c) (i) This work is licensed under a Creative Commons Attribution 4.0 International License. Read Full License

Version of Record: A version of this preprint was published at Breast Cancer Research and Treatment on January 12th, 2022. See the published version at https://doi.org/10.1007/s10549-021-06437-8. 


\section{Abstract \\ Background}

Triple-negative breast cancer (TNBC) is a heterogeneous tumor lacking specific therapeutic targets. Several TNBC classifications have been identified using gene expression profiles, but they are difficult to use in clinical practice. In this study, we have developed a TNBC surrogate subtype classification that represents TNBC subtypes based on the Vanderbilt subtype classification.

\section{Methods}

This study included patients who underwent primary curative breast cancer surgery for TNBC between January 2009 and October 2017 at Seoul St. Mary's Hospital. Representative formalin-fixed paraffin embedded blocks were used for gene expression analysis and tissue microarray construction for immunohistochemical (IHC) staining. The Vanderbilt subtypes were re-classified into 4 groups: basal-like $(B L)$, mesenchymal-like (M), immunomodulatory (IM) and luminal androgen receptor (LAR) subtype. After IHC staining, classification and regression tree (CART) modeling was applied to develop a surrogate subtype classification.

\section{Results}

A total of 145 patients were included in this study. The study cohort was allocated to the Vanderbilt 4 subtypes as LAR $(n=22,15.2 \%), \operatorname{IM}(n=32,22.1 \%), M(n=38,26.2 \%), B L(n=25,17.2 \%)$ and unclassified $(n=28,19.3 \%)$. After excluding nine (6.2\%) patients due to poor IHC staining quality, CART modeling was performed. TNBC surrogate subtypes were defined as follows: LAR subtype, androgen receptor Allred score 8; IM subtype, LAR-negative with a tumor-infiltrating lymphocyte (TIL) score $>70 \%$; M subtype, LARnegative with a TIL score $<20 \%$; BL subtype, LAR-negative with a TIL score $20-70 \%$ and diffuse, strong p16 staining. The study cohort was classified by the surrogate subtypes as LAR $(n=26,17.9 \%), \operatorname{IM}(n=21$, $14.5 \%), M(n=44,30.3 \%), B L 1(n=27,18.6 \%)$ and unclassified $(n=18,12.4 \%)$. The performance of the surrogate subtypes to predict TNBC Vanderbilt 4 subtypes was good with an accuracy of 0.708 . Each subtype exhibited distinct clinicopathologic features, and the M subtype showed a significantly worse survival rate than the other subtypes.

\section{Conclusions}

In this study, we have developed a TNBC surrogate subtype classification that correlates with the Vanderbilt subtype adopting AR, TIL and p16. The surrogate subtype classification is a practical and accessible diagnostic test, that can be easily applied in clinical practice.

\section{Background}


Triple-negative breast cancer (TNBC) is defined by the lack of estrogen receptor and progesterone receptor, along with the absence of HER2 overexpression or amplification. TNBC constitutes $15-20 \%$ of all breast cancers and is characterized by an aggressive clinical course, earlier age of onset, and worse clinical outcomes [1, 2]. Estrogen receptor, progesterone receptor, and HER2 are molecular targets of therapeutic agents that provide improved treatment outcomes in patients with breast cancer. However, patients with TNBC lack targeted therapies and chemotherapy remains the sole established treatment option.

By definition, TNBC is considered a diagnosis of exclusion, which implies that it is a breast cancer subtype comprising heterogeneous tumors [3]. This molecular heterogeneity has resulted in a lack of targeted therapies for TNBC and subsequent failure to achieve improved survival. Thus, a concerted effort is underway to classify this aggressive disease subtype and provide targeted treatment. Several TNBC classifications have been identified based on the results of gene expression profile studies involving unsupervised clustering analyses [4-7]. The TNBC classifications presented in these studies have some common features and subtypes, but do not overlap completely [4-7].

The most commonly used classification method is the Vanderbilt subtype, described by Lehmann and colleagues [4]. Twenty-one publicly available data sets were used to analyze gene expression profiles for 587 TNBC tumors. Six stable molecular subtypes were identified, namely basal-like 1 (BL1), basal-like 2 (BL2), immunomodulatory (IM), luminal androgen receptor (LAR), mesenchymal (M), and mesenchymal stem-like (MSL). In a retrospective study, Masuda et al. demonstrated that the Vanderbilt subtype is an independent predictor of pathologic complete response, implying the potential usefulness of TNBC molecular subtyping in the clinic [8].

TNBC classification based on gene expression profiling is essential for the guidance of individualized treatment. However, gene expression profiling has practical limitations: high cost, a complicated technological process, slow turnaround time, and potential batch effects. These features restrict the application of gene expression-based TNBC subtypes in large clinical trials and routine clinical practice. The identification of a surrogate subtype classification may be an alternative for clinical use because it is much cheaper, faster, and easier to access. In this study, we developed a TNBC surrogate subtype classification that represents TNBC gene expression-based subtypes by using Vanderbilt subtypes.

\section{Methods}

\section{Patient selection and ethical approval}

Patients who underwent breast cancer surgery between January 2009 and October 2017 at Seoul St. Mary's Hospital were included in this retrospective study. Women who were diagnosed with TNBC and underwent primary curative surgery were initially considered eligible for this study. Only patients who provided written informed consent to participate in gene studies were included in this study. Furthermore, patients with a tumor size $<1 \mathrm{~cm}$ and those whose tumors had generated poor-quality formalin-fixed paraffin embedded (FFPE) tissues were excluded to ensure sufficient FFPE tissue for analysis. This study was approved by the institutional review board of Seoul St. Mary's Hospital, College of Medicine, The 
Catholic University of Korea (IRB no. KC17TNSI0414), and the requirement for informed consent was waived.

\section{Tissue microarray construction and RNA microarray expression analysis}

All FFPE sections were reviewed and representative FFPE blocks were marked for RNA extraction and tissue microarray construction. Four 10- $\mu$ m-thick sections were prepared from each representative FFPE block for RNA extraction, and two 2-mm tissue cores per tumor were obtained to construct tissue microarrays. Gene expression analysis was performed using the Affymetrix® Human Gene 2.0 ST Array. The raw data were summarized and normalized with the robust multi-average method implemented in the Affymetrix $®$ Power Tools.

\section{Gene expression-based TNBC subtyping}

The web-based subtyping tool TNBCtype was used to classify the TNBC cohort into Vanderbilt subtypes [9]. The original Vanderbilt subtype method classifies TNBC into six groups, with three main subtypes: basal-like (BL), mesenchymal-like, and LAR [4]. Lehmann et al. refined their TNBC subtypes from six to four subtypes by re-assigning IM and MSL subtypes because these two subtype calls are strongly influenced by stromal cell gene expression [10]. However, considering the importance of immunotherapy in TNBC, maintenance of the IM subtype is needed for precision medicine treatment of TNBC subtypes. Furthermore, TNBC subtypes from other groups include a subtype categorized according to immune response, highlighting the need to maintain the IM subtype $[5-7,11]$. Therefore, we re-classified the Vanderbilt TNBC subtypes into four groups: basal-like (BL), mesenchymal-like (M), immunomodulatory (IM), and luminal androgen receptor (LAR).

\section{Pathology review and immunohistochemical (IHC) staining}

Biomarkers and IHC antibodies for each subtype were selected after a thorough literature review with a preference for antibodies that were already used in pathology clinics to reduce IHC staining bias and provide optimal applicability in clinical practice. p16, EGFR, CK5/6, and p53 were selected for identification of the BL subtype [12-17]. The androgen receptor (AR) was identified as a notable biomarker for the LAR subtype in several previous studies $[4,6,13,16]$. MUC1 overexpression was also reported in LAR subtypes [6]. The IM subtype reportedly shows high expression levels of immune signatures and checkpoint inhibitor genes including PD-L1, tumor-infiltrating lymphocytes (TIL), and CD8 [17]. The M subtype is related to epithelial-mesenchymal transition and is associated with both MUC1 and SMAD4 expression [18, 19].

IHC staining was performed using the tissue microarrays. The details of IHC antibodies are shown in Supplementary Table 1. IHC staining results were assessed in a blinded manner by a pathologist specializing in breast cancer evaluation. AR IHC was evaluated by using the Allred score system [20]. TP53 IHC scoring was based on the proportion of tumor cells with strongly positive nuclear staining, using a grading system of $0 \%, 1-50 \%$, or $>50 \%$. CK5/6 IHC expression was graded with the proportion of tumor cells that showed any cytoplasmic staining ( $<1 \%, 1-50 \%$, or $>50 \%)$. EGFR IHC was graded in accordance with the following system: 0 , no membrane staining; $1+$, faint, partial membrane staining; $2+$, weak, 
complete membrane staining in $>10 \%$ of invasive cancer cells; and $3+$, intense complete membrane staining in $>10 \%$ of invasive cancer cells [21]. p16 IHC expression was graded as negative, weak and mosaic pattern, or diffuse and strong. CD8 scoring was performed from the proportion of CD8-positive lymphocytes in the stromal area with score ranges of $0-10 \%, 11-20 \%, 20-30 \%$, and $>30 \%$. PD-L1 IHC findings were considered positive when PD-L1-positive immune cells were identified in more than $1 \%$ of the tumor area. MUC1 IHC expression was graded on the basis of the proportion of cytoplasmic stained cells with percentage ranges of $0 \%, 1-10 \%, 10-50 \%$, and $>50 \%$ [22]. SMAD4 IHC findings were evaluated according to the staining intensity of any tumor cells using the following categories: negative, weak, or strong [23]. TILs were measured in accordance with recommendations from the International TILs Working Group 2014 [24]. All tumors were evaluated in two cores and summarized by the average values for TILs and CD 8 grading or maximum values for other IHC antibodies.

\section{Statistical analysis}

Clinicopathologic features according to TNBC subtypes were compared using the Pearson chi-squared test or analysis of variance. Disease-free survival was defined as the time from the date of diagnosis to the date of relapse of breast cancer, including locoregional recurrence and/or distant metastasis. Overall survival was defined as the time from the date of diagnosis to patient death or last date of outpatient follow-up. Survival curves were generated using the Kaplan-Meier method and differences in survival according to TNBC subtype were calculated using the log-rank test. Multivariate survival analysis was performed using the Cox proportional hazards regression analysis.

TNBC surrogate subtyping according to pathology review and IHC staining results was performed using the classification and regression trees (CART) model [25]. The predictors included IHC results and TIL value. The target variable was the TNBC Vanderbilt 4 subtype, excluding unclassified patients (these patients were entirely excluded from the modeling process). The parameters of CART were set to max depth $=5$ and complexity parameter $=0.001$. The other parameters were set to default values. The modeling process was performed using the rpart package in R. A novel TNBC surrogate subtype classification was developed based on the CART classification model. The performance of the novel TNBC surrogate subtype classification was estimated by the following parameters: accuracy, specificity, precision, and recall rate. All statistical analysis was performed using $R$ software (R Foundation for Statistical Computing, Vienna, Austria).

\section{Results}

\section{Clinicopathologic characteristics}

In total, 3,158 patients underwent surgical treatment of breast cancer between January 2009 and October 2017. Among them, 485 patients (15.4\%) had TNBC tumors. After exclusion of patients who underwent neoadjuvant chemotherapy, as well as those with very small tumors, insufficient FFPE tissue quality, or lack of informed consent, 147 patients were initially included in this study. Prior to the analysis, two 
additional patients were excluded: one was identified as HER2-positive on additional in situ hybridization tests and one was partially estrogen-receptor-positive.

The clinicopathologic characteristics of the patients with TNBC included in this study are shown in Table 1. The median age at diagnosis was 55 years (range, 18-86 years). The majority of the tumors were invasive ductal carcinoma $(n=111,76.6 \%)$, followed by metaplastic carcinoma $(n=16,11.0 \%)$. The stages of cancer were as follows: stage I, 47 patients (32.4\%); stage II, 87 patients $(60.0 \%)$; and stage III, 11 patients $(7.6 \%)$. 
Table 1

Clinicopathologic characteristics of all patients with TNBC

\begin{tabular}{|lll|}
\hline & & $\mathbf{n}(\%)$ \\
\hline Age & $\leq 35$ & $20(13.8)$ \\
\hline Breast operation & $>35, \leq 50$ & $36(24.8)$ \\
\hline & $>50$ & $89(61.4)$ \\
\hline Axilla operation & BCS & $113(77.9)$ \\
\hline & Mastectomy & $32(22.1)$ \\
\hline Histologic subtype & SLNB & $92(63.4)$ \\
\hline & ALND & $52(35.9)$ \\
\hline Not done & $1(0.7)$ \\
\hline IDCa & ILCa & $111(76.6)$ \\
\hline Histologic grade & Metaplastic & $4(2.8)$ \\
\hline Medullary & $16(11.0)$ \\
\hline Apocrine & $9(6.2)$ \\
\hline Adenoid cystic & $2(1.4)$ \\
\hline Micropapillary & $1(0.7)$ \\
\hline Papillary & $1(0.7)$ \\
\hline Grade 2 & $1(0.7)$ \\
\hline Grade 3 & $23(15.9)$ \\
\hline & No & $122(84.1)$ \\
\hline & Yes cm & $89(61.4)$ \\
\hline
\end{tabular}

BCS, breast-conserving surgery; SLNB, sentinel lymph node biopsy; ALND, axillary lymph node dissection; IDCa, invasive ductal carcinoma; ILCa, invasive lobular carcinoma

${ }^{*}$ Cancer staging was performed in accordance with the AJCC 7th staging system.

${ }^{\dagger}$ Ki67 median value at Seoul St. Mary's Hospital is $25 \%$ 


\begin{tabular}{|c|c|c|}
\hline & & $\mathrm{n}(\%)$ \\
\hline \multirow[t]{3}{*}{$\mathrm{N}$ stage } & NO & $97(66.9)$ \\
\hline & N1 & $36(24.8)$ \\
\hline & N2-3 & $12(8.3)$ \\
\hline \multirow[t]{3}{*}{ Stage ${ }^{*}$} & I & $47(32.4)$ \\
\hline & II & $87(60.0)$ \\
\hline & III & $11(7.6)$ \\
\hline \multirow[t]{2}{*}{$\mathrm{Ki} 67^{\dagger}$} & $<25 \%$ & $11(7.6)$ \\
\hline & $\geq 25 \%$ & $134(92.4)$ \\
\hline \multicolumn{3}{|c|}{$\begin{array}{l}\text { BCS, breast-conserving surgery; SLNB, sentinel lymph node biopsy; ALND, axillary lymph node } \\
\text { dissection; IDCa, invasive ductal carcinoma; ILCa, invasive lobular carcinoma }\end{array}$} \\
\hline \multicolumn{3}{|c|}{${ }^{*}$ Cancer staging was performed in accordance with the AJCC 7th staging system. } \\
\hline \multicolumn{3}{|c|}{${ }^{\dagger}$ Ki67 median value at Seoul St. Mary's Hospital is $25 \%$} \\
\hline
\end{tabular}

The web-based subtyping tool TNBCtype was used to classify the TNBC cohort into Vanderbilt subtypes on the basis of RNA expression data. The cohort was allocated as follows: BL1, 27 patients (18.6\%); BL2, 15 patients (10.3\%); IM, 26 patients (17.9\%); LAR, 15 patients (10.3\%); M, 20 patients (13.8\%); MSL, 17 patients (11.7\%); and unclassified, 25 patients (17.2\%). Vanderbilt subtypes were re-classified into four subtypes (BL, IM, M, and LAR) by re-assigning BL2 and MSL subtypes to the second-highest correlated centroid. After re-classification, the Vanderbilt 4 subtypes were allocated as follows: BL, 25 patients (17.2\%); IM, 32 patients (22.1\%); M, 38 patients (26.2\%); LAR, 22 patients (15.2\%); and unclassified, 28 patients (19.3\%).

TNBC surrogate subtyping was developed using the CART model to correlate with the Vanderbilt 4 subtypes (Fig. 1,2) [25]. Among the 145 patients, nine (6.2\%) were excluded due to poor IHC staining quality. The fitted CART model is illustrated in Supplementary Fig. 1. The original CART model was modified to apply simple and practically feasible criteria for surrogate subtype classification. The LAR subtype constituted an AR Allred score of $8(5+3)$. The IM subtype was defined as LAR-negative findings with a TIL score $>70 \%$. The M subtype was defined as LAR-negative findings with a TIL score $<20 \%$. The BL subtype was defined as LAR-negative, IM-negative, and M-negative findings, with a diffuse and strong p16 staining pattern. The remaining manifestations were considered unclassified. The 136 patients were classified as follows: LAR subtype (26 patients, $17.9 \%$ ), IM subtype (21 patients, $14.5 \%$ ), M subtype (44 patients, 30.3\%), BL1 subtype (27 patients, 18.6\%), and unclassified (18 patients, 12.4\%). Patients 
categorized as unclassified had the $M$ Vanderbilt subtype comprised as the greatest proportion (33.3\%). The unclassified patients from the Vanderbilt 4 subtypes and surrogate subtypes were excluded from the assessment of the performance of the established surrogate classification. The performance of the surrogate subtypes to predict TNBC Vanderbilt 4 subtypes was good (Fig. 3 ). The accuracy was 0.708 , specificity was 0.922 , precision was 0.732 , recall rate was 0.702 , and kappa was 0.608 .

\section{Clinicopathologic features according to TNBC subtypes}

The clinicopathologic features according to Vanderbilt 4 subtypes and surrogate subtypes are compared in Tables 2 and 3 . The mean age of patients with the LAR subtype was significantly older than that of patients with other subtypes. In both classifications, patients with the LAR subtype also had significantly more grade 2 tumors, compared with patients who had the other subtypes. Patients with invasive lobular carcinomas all had the LAR subtype in both classifications, whereas metaplastic carcinomas were mainly present in patients with the M subtype, followed by patients with the LAR subtype. In the Vanderbilt 4 classification, the BL subtype tumor size was significantly larger than tumors of other subtypes. In the surrogate subtype classification, BL subtype tumors were significantly larger, as were unclassified tumors. Axillary lymph node status did not differ in either subtype classification. Low-Ki67 tumors were identified more frequently in patients with the LAR subtype, followed by the M subtype, in both classifications. 
Table 2

Clinicopathologic features according to Vanderbilt 4 subtypes

\begin{tabular}{|c|c|c|c|c|c|c|c|}
\hline & & $\begin{array}{l}\text { LAR (n = } \\
22), n \\
(\%)\end{array}$ & $\begin{array}{l}\operatorname{IM}(n= \\
32), n \\
(\%)\end{array}$ & $\begin{array}{l}M(n= \\
38), n \\
(\%)\end{array}$ & $\begin{array}{l}B L(n= \\
25), n \\
(\%)\end{array}$ & $\begin{array}{l}\text { Unclassified } \\
(n=28), n(\%)\end{array}$ & $p$-value \\
\hline \multirow[t]{4}{*}{ Age } & Mean (SD) & $\begin{array}{l}61.7 \\
(10.4)\end{array}$ & $\begin{array}{l}49.7 \\
(15.5)\end{array}$ & $\begin{array}{l}53.1 \\
(12.7)\end{array}$ & $\begin{array}{l}53.2 \\
(11.4)\end{array}$ & $55.6(11.5)$ & 0.016 \\
\hline & $<35$ & 3 (13.6) & $6(18.8)$ & 7 (18.4) & $1(4.0)$ & $2(7.1)$ & 0.183 \\
\hline & $35-50$ & $\begin{array}{l}17 \\
(77.3)\end{array}$ & $\begin{array}{l}19 \\
(59.4)\end{array}$ & $\begin{array}{l}20 \\
(52.6)\end{array}$ & $\begin{array}{l}13 \\
(52.0)\end{array}$ & $18(64.3)$ & \\
\hline & $>50$ & $2(9.1)$ & $7(21.9)$ & $\begin{array}{l}11 \\
(28.9)\end{array}$ & $\begin{array}{l}11 \\
(44.0)\end{array}$ & 8 (28.6) & \\
\hline \multirow[t]{2}{*}{ Breast Op } & BCS & $\begin{array}{l}12 \\
(54.5)\end{array}$ & $\begin{array}{l}29 \\
(90.6)\end{array}$ & $\begin{array}{l}30 \\
(78.9)\end{array}$ & $\begin{array}{l}19 \\
(76.0)\end{array}$ & $21(75)$ & 0.051 \\
\hline & Mastectomy & $\begin{array}{l}10 \\
(45.5)\end{array}$ & $3(9.4)$ & $8(21.1)$ & $6(24.0)$ & $7(25)$ & \\
\hline \multirow[t]{3}{*}{ Axilla Op } & SLNB & $\begin{array}{l}14 \\
(63.6)\end{array}$ & $\begin{array}{l}18 \\
(56.3)\end{array}$ & $\begin{array}{l}26 \\
(68.4)\end{array}$ & $\begin{array}{l}16 \\
(64.0)\end{array}$ & $18(64.3)$ & 0.948 \\
\hline & ALND & $8(36.4)$ & $\begin{array}{l}13 \\
(40.6)\end{array}$ & $\begin{array}{l}12 \\
(31.6)\end{array}$ & $9(36.0)$ & $10(35.7)$ & \\
\hline & Not done & $0(0)$ & $1(3.1)$ & $0(0)$ & $0(0)$ & $0(0)$ & \\
\hline \multirow[t]{5}{*}{$\begin{array}{l}\text { Histologic } \\
\text { subtype }\end{array}$} & IDCa & $\begin{array}{l}13 \\
(59.1)\end{array}$ & $\begin{array}{l}23 \\
(71.9)\end{array}$ & $\begin{array}{l}29 \\
(76.3)\end{array}$ & $21(84)$ & 25 (89.3) & $<0.001$ \\
\hline & ILCa & 4 (18.2) & $0(0)$ & $0(0)$ & $0(0)$ & $0(0)$ & \\
\hline & Metaplastic & $4(18.2)$ & $1(3.1)$ & $8(21.1)$ & $1(4.0)$ & $2(7.1)$ & \\
\hline & Medullary & $0(0)$ & $7(21.9)$ & $0(0)$ & $1(4.0)$ & $1(3.6)$ & \\
\hline & Others & $1(4.5)$ & $1(3.1)$ & $1(2.6)$ & $2(8.0)$ & $0(0)$ & \\
\hline \multirow[t]{2}{*}{$H G$} & Gr 2 & $9(40.9)$ & $3(9.4)$ & $5(13.2)$ & $2(8.0)$ & $4(14.3)$ & 0.03 \\
\hline & Gr 3 & $\begin{array}{l}13 \\
(59.1)\end{array}$ & $\begin{array}{l}29 \\
(90.6)\end{array}$ & $\begin{array}{l}33 \\
(86.8)\end{array}$ & $\begin{array}{l}23 \\
(92.0)\end{array}$ & $24(85.7)$ & \\
\hline \multicolumn{8}{|c|}{$\begin{array}{l}\text { LAR, luminal androgen receptor; IM, Immunomodulatory; M, mesenchymal-like; BL, basal-like; SD, } \\
\text { standard deviation; BCS, breast-conserving surgery; SLNB, sentinel lymph node biopsy; ALND, axillary } \\
\text { lymph node dissection; IDCa, invasive ductal carcinoma; ILCa, invasive lobular carcinoma; HG, } \\
\text { histology grade; Gr, grade; LVI, lymphovascular invasion }\end{array}$} \\
\hline \multicolumn{8}{|c|}{${ }^{*}$ Cancer staging was performed in accordance with the AJCC 7th staging system. } \\
\hline \multicolumn{8}{|c|}{${ }^{\dagger}$ Ki67 median value at Seoul St. Mary's Hospital is $25 \%$} \\
\hline
\end{tabular}




\begin{tabular}{|c|c|c|c|c|c|c|c|}
\hline & & $\begin{array}{l}\text { LAR (n = } \\
22), n \\
(\%)\end{array}$ & $\begin{array}{l}\operatorname{IM}(n= \\
32), n \\
(\%)\end{array}$ & $\begin{array}{l}M(n= \\
38), n \\
(\%)\end{array}$ & $\begin{array}{l}\mathrm{BL}(\mathrm{n}= \\
25), \mathrm{n} \\
(\%)\end{array}$ & $\begin{array}{l}\text { Unclassified } \\
(n=28), n(\%)\end{array}$ & $p$-value \\
\hline \multirow[t]{2}{*}{ LVI } & No & $\begin{array}{l}13 \\
(59.1)\end{array}$ & $\begin{array}{l}20 \\
(62.5)\end{array}$ & $\begin{array}{l}26 \\
(68.4)\end{array}$ & $\begin{array}{l}14 \\
(56.0)\end{array}$ & 16 (57.1) & 0.848 \\
\hline & Yes & 9 (40.9) & $\begin{array}{l}12 \\
(37.5)\end{array}$ & $\begin{array}{l}12 \\
(31.6)\end{array}$ & $\begin{array}{l}11 \\
(44.0)\end{array}$ & $12(42.9)$ & \\
\hline \multirow[t]{3}{*}{$\begin{array}{l}\text { Tumor } \\
\text { size }\end{array}$} & $\leq 2 \mathrm{~cm}$ & $\begin{array}{l}10 \\
(45.5)\end{array}$ & $\begin{array}{l}18 \\
(56.3)\end{array}$ & $\begin{array}{l}13 \\
(34.2)\end{array}$ & $5(20.0)$ & $14(50)$ & 0.048 \\
\hline & $>2, \leq 5 \mathrm{~cm}$ & $\begin{array}{l}12 \\
(54.5)\end{array}$ & $\begin{array}{l}12 \\
(37.5)\end{array}$ & $\begin{array}{l}24 \\
(63.2)\end{array}$ & $\begin{array}{l}20 \\
(80.0)\end{array}$ & $13(46.4)$ & \\
\hline & $>5 \mathrm{~cm}$ & $0(0)$ & $2(6.3)$ & $1(2.6)$ & $0(0)$ & $1(3.6)$ & \\
\hline \multirow[t]{3}{*}{$\mathrm{N}$ stage } & NO & $\begin{array}{l}15 \\
(68.2)\end{array}$ & $\begin{array}{l}21 \\
(65.6)\end{array}$ & $\begin{array}{l}27 \\
(71.1)\end{array}$ & $\begin{array}{l}16 \\
(64.0)\end{array}$ & $18(64.3)$ & 0.533 \\
\hline & N1 & $7(31.8)$ & 7 (21.9) & $\begin{array}{l}10 \\
(26.3)\end{array}$ & $5(20.0)$ & $6(21.4)$ & \\
\hline & N2-3 & $0(0)$ & $4(12.5)$ & $1(2.6)$ & $4(16.0)$ & $3(10.7)$ & \\
\hline \multirow[t]{3}{*}{ Stage $^{*}$} & 1 & 7 (31.8) & $\begin{array}{l}13 \\
(40.6)\end{array}$ & $\begin{array}{l}13 \\
(34.2)\end{array}$ & $4(16.0)$ & $10(35.7)$ & 0.236 \\
\hline & II & $\begin{array}{l}15 \\
(68.2)\end{array}$ & $\begin{array}{l}16 \\
(50.0)\end{array}$ & $\begin{array}{l}24 \\
(63.2)\end{array}$ & $\begin{array}{l}17 \\
(68.0)\end{array}$ & 15 (53.6) & \\
\hline & III & $0(0)$ & $3(9.4)$ & $1(2.6)$ & $4(16.0)$ & 3 (10.7) & \\
\hline \multirow[t]{2}{*}{$\mathrm{Ki} 67^{+}$} & $<25 \%$ & $6(27.3)$ & $1(3.1)$ & $4(10.5)$ & $0(0)$ & $0(0)$ & 0.002 \\
\hline & $\geq 25 \%$ & $\begin{array}{l}16 \\
(72.7)\end{array}$ & $\begin{array}{l}31 \\
(96.9)\end{array}$ & $\begin{array}{l}34 \\
(89.5)\end{array}$ & $\begin{array}{l}25 \\
(100)\end{array}$ & $28(100)$ & \\
\hline \multirow[t]{2}{*}{ AR } & Negative & $0(0)$ & $\begin{array}{l}10 \\
(31.3)\end{array}$ & $\begin{array}{l}14 \\
(36.8)\end{array}$ & $\begin{array}{l}14 \\
(56.0)\end{array}$ & $9(32.1)$ & $<0.001$ \\
\hline & Positive & $22(100)$ & $\begin{array}{l}22 \\
(68.8)\end{array}$ & $\begin{array}{l}22 \\
(57.9)\end{array}$ & $\begin{array}{l}11 \\
(44.0)\end{array}$ & $16(57.1)$ & $\left(0.275^{\ddagger}\right)$ \\
\hline
\end{tabular}

LAR, luminal androgen receptor; IM, Immunomodulatory; M, mesenchymal-like; BL, basal-like; SD, standard deviation; BCS, breast-conserving surgery; SLNB, sentinel lymph node biopsy; ALND, axillary lymph node dissection; IDCa, invasive ductal carcinoma; ILCa, invasive lobular carcinoma; HG, histology grade; Gr, grade; LVI, lymphovascular invasion

${ }^{*}$ Cancer staging was performed in accordance with the AJCC 7th staging system.

${ }^{+}$Ki67 median value at Seoul St. Mary's Hospital is $25 \%$

${ }^{\ddagger} p$-value when excluding LAR subtype 


\begin{tabular}{|c|c|c|c|c|c|c|c|}
\hline & & $\begin{array}{l}\text { LAR }(n= \\
22), n \\
(\%)\end{array}$ & $\begin{array}{l}\operatorname{IM}(n= \\
32), n \\
(\%)\end{array}$ & $\begin{array}{l}M(n= \\
38), n \\
(\%)\end{array}$ & $\begin{array}{l}\text { BL }(n= \\
25), n \\
(\%)\end{array}$ & $\begin{array}{l}\text { Unclassified } \\
(n=28), n(\%)\end{array}$ & $p$-value \\
\hline \multirow[t]{4}{*}{ CD8 } & $0-10 \%$ & 7 (31.8) & $3(9.4)$ & $\begin{array}{l}16 \\
(42.1)\end{array}$ & $5(20.0)$ & $3(10.7)$ & $<0.001$ \\
\hline & $11-20 \%$ & $8(36.4)$ & $3(9.4)$ & $\begin{array}{l}13 \\
(34.2)\end{array}$ & $5(20.0)$ & $7(25)$ & \\
\hline & $20-30 \%$ & $4(18.2)$ & $\begin{array}{l}11 \\
(34.4)\end{array}$ & $3(7.9)$ & $\begin{array}{l}11 \\
(44.0)\end{array}$ & $7(25)$ & \\
\hline & $>30 \%$ & $1(4.5)$ & $\begin{array}{l}15 \\
(46.9)\end{array}$ & $6(15.8)$ & $4(16.0)$ & $9(32.1)$ & \\
\hline \multirow[t]{3}{*}{ CK5/6 } & Negative & $\begin{array}{l}14 \\
(63.6)\end{array}$ & $\begin{array}{l}11 \\
(34.4)\end{array}$ & $6(15.8)$ & $8(32.0)$ & $13(46.4)$ & 0.001 \\
\hline & Weak & $6(27.3)$ & $\begin{array}{l}10 \\
(31.3)\end{array}$ & $\begin{array}{l}15 \\
(39.5)\end{array}$ & $8(32.0)$ & $7(25)$ & \\
\hline & Strong & $0(0)$ & $\begin{array}{l}10 \\
(31.3)\end{array}$ & $\begin{array}{l}16 \\
(42.1)\end{array}$ & $8(32.0)$ & $4(14.3)$ & \\
\hline \multirow[t]{3}{*}{ EGFR } & Negative & $\begin{array}{l}10 \\
(45.5)\end{array}$ & $6(18.8)$ & $5(13.2)$ & $4(16.0)$ & $4(14.3)$ & 0.022 \\
\hline & Weak & $7(31.8)$ & $\begin{array}{l}14 \\
(43.8)\end{array}$ & $\begin{array}{l}18 \\
(47.4)\end{array}$ & $\begin{array}{l}15 \\
(60.0)\end{array}$ & $17(60.7)$ & \\
\hline & Strong & $2(9.1)$ & $\begin{array}{l}11 \\
(34.4)\end{array}$ & $\begin{array}{l}13 \\
(34.2)\end{array}$ & $5(20.0)$ & $3(10.7)$ & \\
\hline \multirow[t]{4}{*}{ MUC1 } & $0 \%$ & $0(0)$ & $0(0)$ & $5(13.2)$ & $0(0)$ & $0(0)$ & 0.08 \\
\hline & $1-10 \%$ & $2(9.1)$ & $3(9.4)$ & $8(21.1)$ & $3(12.0)$ & $3(10.7)$ & \\
\hline & $10-50 \%$ & $1(4.5)$ & $5(15.6)$ & $8(21.1)$ & $6(24.0)$ & $6(21.4)$ & \\
\hline & $>50 \%$ & $\begin{array}{l}18 \\
(81.8)\end{array}$ & $\begin{array}{l}23 \\
(71.9)\end{array}$ & $\begin{array}{l}17 \\
(44.7)\end{array}$ & $\begin{array}{l}15 \\
(60.0)\end{array}$ & $17(60.7)$ & \\
\hline PD-L1 & Negative & $\begin{array}{l}13 \\
(59.1)\end{array}$ & $2(6.3)$ & $\begin{array}{l}25 \\
(65.8)\end{array}$ & $5(20.0)$ & $6(21.4)$ & $<0.001$ \\
\hline
\end{tabular}

LAR, luminal androgen receptor; IM, Immunomodulatory; M, mesenchymal-like; BL, basal-like; SD, standard deviation; BCS, breast-conserving surgery; SLNB, sentinel lymph node biopsy; ALND, axillary lymph node dissection; IDCa, invasive ductal carcinoma; ILCa, invasive lobular carcinoma; HG, histology grade; Gr, grade; LVI, lymphovascular invasion

${ }^{*}$ Cancer staging was performed in accordance with the AJCC 7th staging system.

${ }^{+}$Ki67 median value at Seoul St. Mary's Hospital is $25 \%$

${ }^{\ddagger}$ p-value when excluding LAR subtype 


\begin{tabular}{|c|c|c|c|c|c|c|c|}
\hline & & $\begin{array}{l}\text { LAR (n = } \\
22), \mathrm{n} \\
(\%)\end{array}$ & $\begin{array}{l}\operatorname{IM}(n= \\
32), n \\
(\%)\end{array}$ & $\begin{array}{l}M(n= \\
38), n \\
(\%)\end{array}$ & $\begin{array}{l}B L(n= \\
25), n \\
(\%)\end{array}$ & $\begin{array}{l}\text { Unclassified } \\
(n=28), n(\%)\end{array}$ & $p$-value \\
\hline & Positive & 9 (40.9) & $\begin{array}{l}30 \\
(93.8)\end{array}$ & $\begin{array}{l}13 \\
(34.2)\end{array}$ & $20(80)$ & 19 (67.9) & \\
\hline \multirow[t]{3}{*}{ p16 } & Negative & $2(9.1)$ & $6(18.8)$ & 7 (18.4) & $2(8)$ & $3(10.7)$ & 0.017 \\
\hline & Mosaic & $\begin{array}{l}12 \\
(54.5)\end{array}$ & $4(12.5)$ & $\begin{array}{l}16 \\
(42.1)\end{array}$ & $5(20)$ & $10(35.7)$ & \\
\hline & Diffuse & $8(36.4)$ & $\begin{array}{l}22 \\
(68.8)\end{array}$ & $\begin{array}{l}15 \\
(39.5)\end{array}$ & $18(72)$ & $14(50)$ & \\
\hline \multirow[t]{3}{*}{ SMAD4 } & Negative & 4 (18.2) & $0(0)$ & $0(0)$ & $1(4.0)$ & $1(3.6)$ & $<0.001$ \\
\hline & Weak & $\begin{array}{l}18 \\
(81.8)\end{array}$ & $\begin{array}{l}10 \\
(31.3)\end{array}$ & $\begin{array}{l}15 \\
(39.5)\end{array}$ & $6(24.0)$ & $12(42.9)$ & \\
\hline & Strong & $0(0)$ & $\begin{array}{l}21 \\
(65.6)\end{array}$ & $\begin{array}{l}23 \\
(60.5)\end{array}$ & $\begin{array}{l}17 \\
(68.0)\end{array}$ & $13(46.4)$ & \\
\hline \multirow[t]{3}{*}{ TP53 } & $0 \%$ & $\begin{array}{l}14 \\
(63.6)\end{array}$ & $\begin{array}{l}11 \\
(34.4)\end{array}$ & $6(15.8)$ & $8(32.0)$ & $13(46.4)$ & $<0.001$ \\
\hline & $1-50 \%$ & 6 (27.3) & $\begin{array}{l}10 \\
(31.3)\end{array}$ & $\begin{array}{l}15 \\
(39.5)\end{array}$ & $8(32.0)$ & $7(25)$ & \\
\hline & $>50 \%$ & $0(0)$ & $\begin{array}{l}10 \\
(31.3)\end{array}$ & $\begin{array}{l}16 \\
(42.1)\end{array}$ & $8(32.0)$ & $4(14.3)$ & \\
\hline \multirow[t]{4}{*}{ TIL } & $0-15 \%$ & $\begin{array}{l}13 \\
(59.1)\end{array}$ & $2(6.3)$ & $\begin{array}{l}30 \\
(78.9)\end{array}$ & $7(28.0)$ & $6(21.4)$ & $<0.001$ \\
\hline & $15-45 \%$ & 4 (18.2) & $4(12.5)$ & $2(5.3)$ & $8(32.0)$ & $7(25)$ & \\
\hline & $45-75 \%$ & 4 (18.2) & $\begin{array}{l}12 \\
(37.5)\end{array}$ & $4(10.5)$ & $8(32.0)$ & $4(14.3)$ & \\
\hline & $75-100 \%$ & $0(0)$ & $\begin{array}{l}13 \\
(40.6)\end{array}$ & $0(0)$ & $1(4.0)$ & $9(32.1)$ & \\
\hline \multicolumn{8}{|c|}{$\begin{array}{l}\text { LAR, luminal androgen receptor; IM, Immunomodulatory; M, mesenchymal-like; BL, basal-like; SD, } \\
\text { standard deviation; BCS, breast-conserving surgery; SLNB, sentinel lymph node biopsy; ALND, axillary } \\
\text { lymph node dissection; IDCa, invasive ductal carcinoma; ILCa, invasive lobular carcinoma; HG, } \\
\text { histology grade; Gr, grade; LVI, lymphovascular invasion }\end{array}$} \\
\hline \multicolumn{8}{|c|}{${ }^{*}$ Cancer staging was performed in accordance with the AJCC 7th staging system. } \\
\hline \multicolumn{8}{|c|}{${ }^{\dagger}$ Ki67 median value at Seoul St. Mary's Hospital is $25 \%$} \\
\hline
\end{tabular}


Table 3

Clinicopathologic features according to the TNBC surrogate subtype classification

\begin{tabular}{|c|c|c|c|c|c|c|c|}
\hline & & $\begin{array}{l}\text { LAR (n = } \\
26), n \\
(\%)\end{array}$ & $\begin{array}{l}\operatorname{IM}(n= \\
21), n \\
(\%)\end{array}$ & $\begin{array}{l}M(n= \\
44), n \\
(\%)\end{array}$ & $\begin{array}{l}\text { BL }(n= \\
27), n \\
(\%)\end{array}$ & $\begin{array}{l}\text { Unclassified } \\
(n=18), n(\%)\end{array}$ & $p$-value \\
\hline \multirow[t]{4}{*}{ Age } & Mean (SD) & $\begin{array}{l}61.3 \\
(12.3)\end{array}$ & $\begin{array}{l}50.1 \\
(13.2)\end{array}$ & $\begin{array}{l}54.4 \\
(12.3)\end{array}$ & $\begin{array}{l}51.7 \\
(12.1)\end{array}$ & $50.8(12.2)$ & 0.012 \\
\hline & $<35$ & 5 (19.2) & $2(9.5)$ & $5(11.4)$ & $2(7.4)$ & $2(11.1)$ & 0.292 \\
\hline & $35-50$ & $\begin{array}{l}19 \\
(73.1)\end{array}$ & $\begin{array}{l}11 \\
(52.4)\end{array}$ & $\begin{array}{l}27 \\
(61.4)\end{array}$ & $\begin{array}{l}16 \\
(59.3)\end{array}$ & $9(50.0)$ & \\
\hline & $>50$ & $2(7.7)$ & 8 (38.1) & $\begin{array}{l}12 \\
(27.3)\end{array}$ & $9(33.3)$ & 7 (38.9) & \\
\hline \multirow[t]{2}{*}{ Breast Op } & BCS & $\begin{array}{l}17 \\
(65.4)\end{array}$ & $\begin{array}{l}19 \\
(90.5)\end{array}$ & $\begin{array}{l}33 \\
(75.0)\end{array}$ & $\begin{array}{l}21 \\
(77.8)\end{array}$ & $14(77.8)$ & 0.388 \\
\hline & Mastectomy & $9(34.6)$ & $2(9.5)$ & $\begin{array}{l}11 \\
(25.0)\end{array}$ & $6(22.2)$ & $4(22.2)$ & \\
\hline \multirow[t]{3}{*}{ Axilla Op } & SLNB & $\begin{array}{l}17 \\
(65.4)\end{array}$ & $\begin{array}{l}11 \\
(52.4)\end{array}$ & $\begin{array}{l}30 \\
(68.2)\end{array}$ & $\begin{array}{l}16 \\
(59.3)\end{array}$ & $11(61.1)$ & 0.706 \\
\hline & ALND & $8(30.8)$ & $\begin{array}{l}10 \\
(47.6)\end{array}$ & $\begin{array}{l}14 \\
(31.8)\end{array}$ & $\begin{array}{l}11 \\
(40.7)\end{array}$ & 7 (38.9) & \\
\hline & Not done & $1(3.8)$ & $0(0)$ & $0(0)$ & $0(0)$ & $0(0)$ & \\
\hline \multirow[t]{5}{*}{$\begin{array}{l}\text { Histologic } \\
\text { subtype }\end{array}$} & IDCa & $\begin{array}{l}17 \\
(65.4)\end{array}$ & $\begin{array}{l}15 \\
(71.4)\end{array}$ & $\begin{array}{l}33 \\
(75.0)\end{array}$ & $\begin{array}{l}24 \\
(88.9)\end{array}$ & $16(88.9)$ & 0.014 \\
\hline & ILCa & $4(15.4)$ & $0(0)$ & $0(0)$ & $0(0)$ & $0(0)$ & \\
\hline & Metaplastic & $3(11.5)$ & $0(0)$ & $7(15.9)$ & $2(7.4)$ & $1(5.6)$ & \\
\hline & Medullary & $1(3.8)$ & $5(23.8)$ & $1(2.3)$ & $1(3.7)$ & $1(5.6)$ & \\
\hline & Others & $1(3.8)$ & $1(4.8)$ & $3(6.8)$ & $0(0)$ & $0(0)$ & \\
\hline $\mathrm{HG}$ & Gr 2 & $\begin{array}{l}10 \\
(38.5)\end{array}$ & $1(4.8)$ & $6(13.6)$ & $1(3.7)$ & 3 (16.7) & 0.006 \\
\hline
\end{tabular}

LAR, luminal androgen receptor; IM, Immunomodulatory; M, mesenchymal-like; BL, basal-like; SD, standard deviation; BCS, breast-conserving surgery; SLNB, sentinel lymph node biopsy; ALND, axillary lymph node dissection; IDCa, invasive ductal carcinoma; ILCa, invasive lobular carcinoma; HG, histology grade; Gr, grade; LVI, lymphovascular invasion

${ }^{*}$ Cancer staging was performed in accordance with the AJCC 7th staging system.

${ }^{+}$Ki67 median value at Seoul St. Mary's Hospital is $25 \%$

${ }^{\ddagger}$ p-value when excluding LAR subtype 


\begin{tabular}{|c|c|c|c|c|c|c|c|}
\hline & & $\begin{array}{l}\text { LAR }(\mathrm{n}= \\
26), \mathrm{n} \\
(\%)\end{array}$ & $\begin{array}{l}\operatorname{IM}(n= \\
21), n \\
(\%)\end{array}$ & $\begin{array}{l}M(n= \\
44), n \\
(\%)\end{array}$ & $\begin{array}{l}\text { BL }(n= \\
27), n \\
(\%)\end{array}$ & $\begin{array}{l}\text { Unclassified } \\
(n=18), n(\%)\end{array}$ & $p$-value \\
\hline & Gr 3 & $\begin{array}{l}16 \\
(61.5)\end{array}$ & $\begin{array}{l}20 \\
(95.2)\end{array}$ & $\begin{array}{l}38 \\
(86.4)\end{array}$ & $\begin{array}{l}26 \\
(96.3)\end{array}$ & $15(83.3)$ & \\
\hline \multirow[t]{2}{*}{ LVI } & No & $\begin{array}{l}17 \\
(65.4)\end{array}$ & $\begin{array}{l}13 \\
(61.9)\end{array}$ & $\begin{array}{l}25 \\
(56.8)\end{array}$ & $\begin{array}{l}17 \\
(63.0)\end{array}$ & $11(61.1)$ & 0.965 \\
\hline & Yes & $9(34.6)$ & $8(38.1)$ & $\begin{array}{l}19 \\
(43.2)\end{array}$ & $10(37)$ & 7 (38.9) & \\
\hline \multirow[t]{3}{*}{$\begin{array}{l}\text { Tumor } \\
\text { size }\end{array}$} & $\leq 2 \mathrm{~cm}$ & $\begin{array}{l}12 \\
(46.2)\end{array}$ & $\begin{array}{l}15 \\
(71.4)\end{array}$ & $\begin{array}{l}18 \\
(40.9)\end{array}$ & $8(29.6)$ & $3(16.7)$ & 0.018 \\
\hline & $>2, \leq 5 \mathrm{~cm}$ & $\begin{array}{l}13 \\
(50.0)\end{array}$ & $6(28.6)$ & $\begin{array}{l}25 \\
(56.8)\end{array}$ & $\begin{array}{l}18 \\
(66.7)\end{array}$ & $14(77.8)$ & \\
\hline & $>5 \mathrm{~cm}$ & $1(3.8)$ & $0(0)$ & $1(2.3)$ & $1(3.7)$ & $1(5.6)$ & \\
\hline \multirow[t]{3}{*}{ N stage } & NO & $\begin{array}{l}17 \\
(65.4)\end{array}$ & $\begin{array}{l}13 \\
(61.9)\end{array}$ & $\begin{array}{l}33 \\
(75.0)\end{array}$ & $\begin{array}{l}16 \\
(59.3)\end{array}$ & $11(61.1)$ & 0.455 \\
\hline & N1 & $7(26.9)$ & 7 (33.3) & $9(20.5)$ & $8(29.6)$ & $3(16.7)$ & \\
\hline & N2-3 & $1(3.8)$ & $1(4.8)$ & $2(4.5)$ & $3(11.1)$ & $4(22.2)$ & \\
\hline \multirow[t]{3}{*}{ Stage $^{*}$} & I & 9 (34.6) & $9(42.9)$ & $\begin{array}{l}17 \\
(38.6)\end{array}$ & $6(22.2)$ & $3(16.7)$ & 0.288 \\
\hline & II & $\begin{array}{l}16 \\
(61.5)\end{array}$ & $\begin{array}{l}11 \\
(52.4)\end{array}$ & $\begin{array}{l}25 \\
(56.8)\end{array}$ & $\begin{array}{l}19 \\
(70.4)\end{array}$ & $11(61.1)$ & \\
\hline & III & $1(3.8)$ & $1(4.8)$ & $2(4.5)$ & $2(7.4)$ & $4(22.2)$ & \\
\hline \multirow[t]{2}{*}{$\mathrm{Ki} 67^{\dagger}$} & $<25 \%$ & 7 (26.9) & $0(0)$ & $2(4.5)$ & $0(0)$ & $2(11.1)$ & 0.002 \\
\hline & $\geq 25 \%$ & $\begin{array}{l}19 \\
(73.1)\end{array}$ & $\begin{array}{l}21 \\
(100)\end{array}$ & $\begin{array}{l}42 \\
(95.5)\end{array}$ & $\begin{array}{l}27 \\
(100)\end{array}$ & $16(88.9)$ & \\
\hline \multirow[t]{2}{*}{ AR } & Negative & $0(0)$ & $9(42.9)$ & $\begin{array}{l}19 \\
(43.2)\end{array}$ & $\begin{array}{l}12 \\
(44.4)\end{array}$ & 6 (33.3) & $<0.001$ \\
\hline & Positive & $26(100)$ & $\begin{array}{l}12 \\
(57.1)\end{array}$ & $\begin{array}{l}25 \\
(56.8)\end{array}$ & $\begin{array}{l}15 \\
(55.6)\end{array}$ & $12(66.7)$ & $\left(0.884^{\ddagger}\right)$ \\
\hline \multicolumn{8}{|c|}{$\begin{array}{l}\text { LAR, luminal androgen receptor; IM, Immunomodulatory; M, mesenchymal-like; BL, basal-like; SD, } \\
\text { standard deviation; BCS, breast-conserving surgery; SLNB, sentinel lymph node biopsy; ALND, axillary } \\
\text { lymph node dissection; IDCa, invasive ductal carcinoma; ILCa, invasive lobular carcinoma; HG, } \\
\text { histology grade; Gr, grade; LVI, lymphovascular invasion }\end{array}$} \\
\hline \multicolumn{8}{|c|}{${ }^{*}$ Cancer staging was performed in accordance with the AJCC 7th staging system. } \\
\hline \multicolumn{8}{|c|}{${ }^{\dagger}$ Ki67 median value at Seoul St. Mary's Hospital is $25 \%$} \\
\hline \multicolumn{8}{|c|}{${ }^{\ddagger} \mathrm{p}$-value when excluding LAR subtype } \\
\hline
\end{tabular}




\begin{tabular}{|c|c|c|c|c|c|c|c|}
\hline & & $\begin{array}{l}\text { LAR (n = } \\
26), n \\
(\%)\end{array}$ & $\begin{array}{l}\operatorname{IM}(n= \\
21), n \\
(\%)\end{array}$ & $\begin{array}{l}M(n= \\
44), n \\
(\%)\end{array}$ & $\begin{array}{l}\text { BL }(n= \\
27), n \\
(\%)\end{array}$ & $\begin{array}{l}\text { Unclassified } \\
(n=18), n(\%)\end{array}$ & $p$-value \\
\hline \multirow[t]{4}{*}{ CD8 } & $0-10 \%$ & $6(23.1)$ & $0(0)$ & $22(50)$ & $3(11.1)$ & $1(5.6)$ & $<0.001$ \\
\hline & $11-20 \%$ & $8(30.8)$ & $2(9.5)$ & $\begin{array}{l}16 \\
(36.4)\end{array}$ & $5(18.5)$ & $2(11.1)$ & \\
\hline & $20-30 \%$ & $7(26.9)$ & $2(9.5)$ & $4(9.1)$ & $\begin{array}{l}12 \\
(44.4)\end{array}$ & $10(55.6)$ & \\
\hline & $>30 \%$ & $3(11.5)$ & $\begin{array}{l}17 \\
(81.0)\end{array}$ & $2(4.5)$ & $7(25.9)$ & $5(27.8)$ & \\
\hline \multirow[t]{3}{*}{ CK5/6 } & Negative & $\begin{array}{l}16 \\
(61.5)\end{array}$ & $\begin{array}{l}10 \\
(47.6)\end{array}$ & $\begin{array}{l}11 \\
(25.0)\end{array}$ & $7(25.9)$ & $6(33.3)$ & 0.003 \\
\hline & Weak & 7 (26.9) & 7 (33.3) & $\begin{array}{l}16 \\
(36.4)\end{array}$ & $\begin{array}{l}11 \\
(40.7)\end{array}$ & $4(22.2)$ & \\
\hline & Strong & $0(0)$ & $4(19.0)$ & $\begin{array}{l}15 \\
(34.1)\end{array}$ & $9(33.3)$ & $8(44.4)$ & \\
\hline \multirow[t]{3}{*}{ EGFR } & Negative & $\begin{array}{l}12 \\
(46.2)\end{array}$ & $5(23.8)$ & 8 (18.2) & $3(11.1)$ & $1(5.6)$ & 0.05 \\
\hline & Weak & $9(34.6)$ & $\begin{array}{l}12 \\
(57.1)\end{array}$ & $\begin{array}{l}22 \\
(50.0)\end{array}$ & $\begin{array}{l}17 \\
(63.0)\end{array}$ & $8(44.4)$ & \\
\hline & Strong & $4(15.4)$ & $4(19)$ & 9 (20.5) & $7(25.9)$ & $8(44.4)$ & \\
\hline \multirow[t]{4}{*}{ MUC1 } & $0 \%$ & $0(0)$ & $0(0)$ & $3(6.8)$ & $0(0)$ & $0(0)$ & 0.602 \\
\hline & $1-10 \%$ & $2(7.7)$ & $1(4.8)$ & $9(20.5)$ & $3(11.1)$ & $3(16.7)$ & \\
\hline & $10-50 \%$ & $4(15.4)$ & $3(14.3)$ & $7(15.9)$ & $7(25.9)$ & $4(22.2)$ & \\
\hline & $>50 \%$ & $\begin{array}{l}19 \\
(73.1)\end{array}$ & $17(81)$ & $\begin{array}{l}25 \\
(56.8)\end{array}$ & $17(63)$ & $11(61.1)$ & \\
\hline \multirow[t]{2}{*}{ PD-L1 } & Negative & $13(50)$ & $0(0)$ & $33(75)$ & $3(11.1)$ & $0(0)$ & $<0.001$ \\
\hline & Positive & $13(50)$ & $\begin{array}{l}21 \\
(100)\end{array}$ & 110 & $\begin{array}{l}24 \\
(88.9)\end{array}$ & $18(100)$ & \\
\hline
\end{tabular}

LAR, luminal androgen receptor; IM, Immunomodulatory; M, mesenchymal-like; BL, basal-like; SD, standard deviation; BCS, breast-conserving surgery; SLNB, sentinel lymph node biopsy; ALND, axillary lymph node dissection; IDCa, invasive ductal carcinoma; ILCa, invasive lobular carcinoma; HG, histology grade; Gr, grade; LVI, lymphovascular invasion

${ }^{*}$ Cancer staging was performed in accordance with the AJCC 7th staging system.

${ }^{+}$Ki67 median value at Seoul St. Mary's Hospital is $25 \%$

${ }^{\ddagger}$ p-value when excluding LAR subtype 


\begin{tabular}{|c|c|c|c|c|c|c|c|}
\hline & & $\begin{array}{l}\text { LAR (n = } \\
26), n \\
(\%)\end{array}$ & $\begin{array}{l}\operatorname{IM}(n= \\
21), n \\
(\%)\end{array}$ & $\begin{array}{l}M(n= \\
44), n \\
(\%)\end{array}$ & $\begin{array}{l}\text { BL }(n= \\
27), n \\
(\%)\end{array}$ & $\begin{array}{l}\text { Unclassified } \\
(n=18), n(\%)\end{array}$ & $p$-value \\
\hline \multirow[t]{3}{*}{ p16 } & Negative & $1(3.8)$ & $4(19)$ & 6 (13.6) & $0(0.0)$ & $8(44.4)$ & $<0.001$ \\
\hline & Mosaic & $\begin{array}{l}16 \\
(61.5)\end{array}$ & $2(9.5)$ & $\begin{array}{l}16 \\
(36.4)\end{array}$ & $0(0.0)$ & $10(55.6)$ & \\
\hline & Diffuse & 9 (34.6) & $\begin{array}{l}15 \\
(71.4)\end{array}$ & $\begin{array}{l}22 \\
(50.0)\end{array}$ & $\begin{array}{l}27 \\
(100)\end{array}$ & $0(0.0)$ & \\
\hline \multirow[t]{3}{*}{ SMAD4 } & Negative & $1(3.8)$ & $0(0)$ & $3(6.8)$ & $2(7.4)$ & $0(0)$ & 0.01 \\
\hline & Weak & $\begin{array}{l}19 \\
(73.1)\end{array}$ & $6(28.6)$ & $\begin{array}{l}19 \\
(43.2)\end{array}$ & $7(25.9)$ & $8(44.4)$ & \\
\hline & Strong & $6(23.1)$ & $\begin{array}{l}15 \\
(71.4)\end{array}$ & $\begin{array}{l}22 \\
(50.0)\end{array}$ & $\begin{array}{l}18 \\
(66.7)\end{array}$ & $10(55.6)$ & \\
\hline \multirow[t]{3}{*}{ TP53 } & $0 \%$ & $\begin{array}{l}16 \\
(61.5)\end{array}$ & $\begin{array}{l}10 \\
(47.6)\end{array}$ & $\begin{array}{l}11 \\
(25.0)\end{array}$ & $7(25.9)$ & $6(33.3)$ & 0.002 \\
\hline & $1-50 \%$ & 7 (26.9) & $7(33.3)$ & $\begin{array}{l}16 \\
(36.4)\end{array}$ & $\begin{array}{l}11 \\
(40.7)\end{array}$ & 4 (22.2) & \\
\hline & $>50 \%$ & $0(0)$ & $4(19.0)$ & $\begin{array}{l}15 \\
(34.1)\end{array}$ & $9(33.3)$ & $8(44.4)$ & \\
\hline \multirow[t]{4}{*}{ TIL } & $0-15 \%$ & $\begin{array}{l}12 \\
(46.2)\end{array}$ & $0(0)$ & $\begin{array}{l}44 \\
(100)\end{array}$ & $0(0)$ & $0(0)$ & $<0.001$ \\
\hline & $15-45 \%$ & $4(15.4)$ & $0(0)$ & $0(0)$ & $\begin{array}{l}13 \\
(48.1)\end{array}$ & $8(44.4)$ & \\
\hline & $45-75 \%$ & $6(23.1)$ & $2(9.5)$ & $0(0)$ & $\begin{array}{l}14 \\
(51.9)\end{array}$ & $10(55.6)$ & \\
\hline & $75-100 \%$ & 3 (11.5) & $\begin{array}{l}19 \\
(90.5)\end{array}$ & $0(0)$ & $0(0)$ & $0(0)$ & \\
\hline \multicolumn{8}{|c|}{$\begin{array}{l}\text { LAR, luminal androgen receptor; IM, Immunomodulatory; M, mesenchymal-like; BL, basal-like; SD, } \\
\text { standard deviation; BCS, breast-conserving surgery; SLNB, sentinel lymph node biopsy; ALND, axillary } \\
\text { lymph node dissection; IDCa, invasive ductal carcinoma; ILCa, invasive lobular carcinoma; HG, } \\
\text { histology grade; Gr, grade; LVI, lymphovascular invasion }\end{array}$} \\
\hline \multicolumn{8}{|c|}{${ }^{*}$ Cancer staging was performed in accordance with the AJCC 7th staging system. } \\
\hline \multicolumn{8}{|c|}{${ }^{\dagger}$ Ki67 median value at Seoul St. Mary's Hospital is $25 \%$} \\
\hline \multicolumn{8}{|c|}{${ }^{\ddagger} \mathrm{p}$-value when excluding LAR subtype } \\
\hline
\end{tabular}

Similar characteristics related to the IHC results of biomarkers not included in the surrogate subtype classification were also identified in both classifications (Tables 2 and 3). A high PD-L1-positive rate was identified among patients with the IM and BL1 subtypes. Notably, all IM tumors from the surrogate 
classification exhibited with PD-L1-positive staining. EGFR and CK5/6 showed negative or weak expression among patients with the LAR subtype. Whereas EGFR and CK5/ 6 did not show any specific pattern among patients with the BL subtype, compared with patients who had the IM or M subtypes. SMAD4 and TP53 also showed negative or weak expression among patients with the LAR subtype.

The median follow-up duration was 41 months (range, 0-64 months). During the study period, 25 patients (17.2\%) experienced locoregional recurrence or distant metastasis. Disease-free survival did not differ according to the Vanderbilt 4 subtypes, but a tendency for worse survival was noted among patients with the M subtype ( $p=0.16$, Fig. 4$)$. When classified according to surrogate subtypes, patients with the $M$ subtype demonstrated significantly worse disease-free survival ( $p=0.004$, Fig. 2$)$. M subtype persisted as an independent risk factor for poor survival after adjusting for other prognostic factors (HR $11.401 ; 95 \% \mathrm{Cl}$ 1.488-87.370; $p=0.019$; Table 4). Although it was not statistically significant, a tendency for better survival among patients with the IM subtype was also identified in both classifications. No difference in overall survival was noted in either classification (Vanderbilt 4 subtypes overall survival, $p=0.238$; surrogate subtypes overall survival, $p=0.061$; Fig. 4 ).

Table 4

Univariate and multivariate analysis of prognostic factors and TNBC surrogate subtype classification

\begin{tabular}{|c|c|c|c|c|c|c|c|c|c|}
\hline & & \multicolumn{4}{|c|}{ Univariate } & \multicolumn{4}{|c|}{ Multivariate } \\
\hline & & $\mathrm{HR}$ & $95 \% \mathrm{Cl}$ & & $\begin{array}{l}p- \\
\text { value }\end{array}$ & $\mathrm{HR}$ & $95 \% \mathrm{Cl}$ & & $\begin{array}{l}p- \\
\text { value }\end{array}$ \\
\hline Age & continuous & 1.046 & 1.010 & 1.083 & 0.011 & 1.061 & 1.022 & 1.104 & 0.002 \\
\hline HG & Gr 3 vs Gr 2 & 0.984 & 0.338 & 2.869 & 0.977 & & & & \\
\hline LVI & Yes vs No & 1.574 & 0.718 & 3.455 & 0.258 & & & & \\
\hline $\begin{array}{l}\text { Tumor } \\
\text { size }\end{array}$ & continuous & 1.201 & 0.933 & 1.547 & 0.156 & & & & \\
\hline N stage & $\mathrm{N}(+)$ vs NO & 2.455 & 1.120 & 5.381 & 0.026 & 2.632 & 1.170 & 5.924 & 0.019 \\
\hline Ki67 (\%) & continuous & 1.833 & 0.248 & 13.570 & 0.553 & & & & \\
\hline \multirow{5}{*}{$\begin{array}{l}\text { TNBC } \\
\text { surrogate } \\
\text { subtype }\end{array}$} & IM & \multicolumn{4}{|c|}{ Reference } & \multicolumn{4}{|c|}{ Reference } \\
\hline & LAR & 4.029 & 0.450 & 36.100 & 0.213 & 2.716 & 0.297 & 24.863 & 0.376 \\
\hline & M & 10.100 & 1.331 & 76.670 & 0.025 & 11.401 & 1.488 & 87.370 & 0.019 \\
\hline & $B L$ & 2.969 & 0.309 & 28.560 & 0.346 & 3.315 & 0.343 & 32.049 & 0.301 \\
\hline & Unclassified & 1.231 & 0.077 & 19.670 & 0.883 & 1.367 & 0.085 & 21.921 & 0.825 \\
\hline
\end{tabular}


TNBC is a heterogeneous disease and subtype classification is necessary for optimization of personalized treatment. TNBC subtyping using gene expression data has been implemented by several groups but gene expression data are impractical and expensive for clinical application. To aid in this subtyping effort, we developed a TNBC surrogate subtype classification to classify TNBC into four subtypes that correlate with the Vanderbilt subtypes. The TNBC surrogate subtyping adopts $\mathrm{p} 16, \mathrm{AR}$, and TIL data for classification, which are widely used parameters in pathology clinics.

This is the first study to develop a TNBC surrogate subtype that correlates with the Vanderbilt subtypes. Zhao et al. developed an IHC-based approach for TNBC subtyping by comparison with mRNA-based subtypes used in the Fudan University Shanghai Cancer Center [26]. Other similar studies have attempted to classify TNBC subtypes by IHC panels but have focused on independent classifications that did not involve correlations with any gene expression data $[13,16]$.

The LAR subtype is universally identified among TNBC gene expression subtypes that use unsupervised clustering methods [4-7]. Notably, the LAR subtype is enriched in AR expression and its downstream gene targets and is relatively simple to identify using AR protein expression in $\mathrm{IHC}$ analysis $[4,27,28]$. In this study, we used an AR Allred score of 8 to identify the LAR subtype. This comprised $18 \%$ of the TNBC cohort, similar to previous reports in which $10-35 \%$ of TNBC tumors expressed AR [29-32]. The clinical significance of AR expression has been identified in several clinical trials, indicating the efficacy of antiandrogen treatment in patients with AR-positive metastatic TNBC [33, 34]. Clinical trials of anti-androgens have used cutoff values of $1 \%$ or $10 \%$, which differ from our study. The predictive value of AR expression levels for anti-androgen treatment requires further investigation, but the present study implies that a higher cutoff value may be needed to identify a LAR subtype more specifically in patients with TNBC.

Another subtype consistently identified in distinct gene expression classification systems is the IM subtype $[4,6,7,11]$. Gene expression data have shown that this subtype is highly enriched for immune cell signaling, and that it has a high prevalence of TILs $[10,11]$. This correlates with the use of TIL as a marker to determine IM subtype in the surrogate subtype classification. The clinical implications of the IM subtype and TIL as a subtype marker are related to the use of immune checkpoint inhibitor therapies, which are currently a novel component of care for patients with TNBC [35]. Atezolizumab, a PD-L1 inhibitor, has been approved in combination with nab-paclitaxel for PD-L1-positive metastatic TNBCs and TIL is a predictive factor when tumors are PD-L1 positive $[36,37]$. The predictive value of TILs has also been identified in clinical trials for pembrolizumab, a PD-1 inhibitor [38, 39]. In this study, both IM subtypes classified on the basis of RNA and IHC expression exhibited high CD8 + levels and high rates of PD-L1 positivity, which are additional biomarkers that serve as predictive factors for immune checkpoint inhibitors [40].

In contrast to the IM subtype, low TIL ( $<20 \%)$ was the determining factor for the M subtype in the IHC classification. Lehmann et al. reported that $\mathrm{M}$ and IM subtypes had a negative correlation, which was consistent with the classification method in our study [10]. The negative correlation between the $M$ and IM subtypes implies that the $M$ subtype involves an immunosuppressed microenvironment, corresponding to the poor prognosis of patients with the $M$ subtype [10]. The clinical significance of the $M$ subtype as a target for novel therapeutics is unclear. Bioinformatics analysis of data derived from the Molecular 
Taxonomy of Breast Cancer International Consortium (METABRIC) and The Cancer Genome Atlas (TCGA) showed that the $M$ subtype is associated with an elevated angiogenesis signature score and enrichment of the EGFR and Notch signaling pathways [17]. On the basis of these results, antiangiogenic therapy or EGFR inhibitors could be considered for patients with M subtype tumors, which have both failed to demonstrate a survival benefit in unselected TNBC populations $[41,42]$.

Immunostainings for basal cytokeratins including CK5/6, CK14, CK17, and EGFR have been regarded as surrogate markers of the $B L$ subtype. However diffuse and strong staining of p16 immunostaining is the last condition of BL subtype in our TNBC surrogate subtype classification. This correlates with the TNBC cohort from the METABRIC and TCGA dataset, showing copy number amplifications in CDKN2A/B genes in the BL1 subtype [17]. p16 regulates the cell cycle by inhibition of phosphorylation of $R b$ via inactivation of CDK4/6 [43]. The positive immunostaining of p16 is suggested as a surrogate marker for Rb pathway loss, and $\mathrm{Rb} 1$ loss is associated with homologous recombination deficiency in high-grade serous ovarian cancer $[45,46]$. This suggests that adopting p16 immunostaining as a surrogate marker for BL subtype in our TNBC surrogate subtype classification is related to Rb1 loss and homologous recombination deficiency. The BL subtype itself is characterized by high genomic instability and high copy number losses for BRCA1/2, which implies sensitivity to poly-ADP ribose polymerase(PARP) inhibitors. PARP inhibitors have shown considerable benefit in patients with advanced breast cancer and a germline BRCA1/2 mutation, especially those with TNBC tumors $[48,49]$. Currently, the presence of germline BRCA $1 / 2$ mutations is the main predictive factor for the effects of PARP inhibitors, but the BL subtype could also be regarded as a predictive factor, especially in patients with wild-type BRCA 1/2 with homologous recombination deficiency.

The strength of the TNBC surrogate subtype classification is that AR, p16 IHC, and TIL evaluation protocols are widely used in pathology clinics. Guidelines for IHC procedures and TIL assessment are already established [20,24], so the clinical application of the surrogate subtype classification is easy and immediately available.

However, there were some weaknesses in this study. Tissue microarrays were used for TIL evaluation and IHC staining, which may not represent the entire tumor. RNA sequencing was performed using bulk tumor tissue, which does not reflect the intratumoral heterogeneity demonstrated by single-cell RNA sequencing [50]. Eighteen patients (13.2\%) were unclassified in the surrogate subtype classification and could not be included in any specific Vanderbilt 4 subtype. This lack of classification requires further investigation. CART is a relatively unstable method, whereby a small change in the data can cause considerable variation in the model, especially when a small sample size is used [51]. The TNBC cohort in this study was relatively small and further validation is needed using a larger, external dataset. Also, all patients included in this study underwent primary surgery followed by adjuvant chemotherapy, which is deviated from current treatment guidelines that recommend preoperative systemic therapy in patients with TNBC tumors. An additional study using core needle biopsy tissues in patients undergoing neoadjuvant therapy is needed to confirm the application of the surrogate subtypes in current TNBC treatment. 


\section{Conclusions}

In this study, we developed a novel TNBC surrogate subtype classification that correlates with the gene expression classification established by the Vanderbilt group. The surrogate subtype adopts AR, TIL, and p16, thereby classifying TNBC with a practical and easily accessible diagnostic test. Each subtype exhibits diverse clinicopathological and prognostic characteristics. Further studies are needed to validate the clinical utility of the surrogate subtype classification for use in personalized treatment with targeted and immune-based therapies.

\section{Abbreviations}

AR

Androgen receptor

BL

Basal-like

CART

Classification and regression tree

FFPE

Formalin-fixed paraffine embedded

IHC

Immunohistochemical

IM

Immunomodulatory

LAR

Luminal androgen receptor

$\mathrm{M}$

Mesenchymal-like

METABRIC

Molecular Taxonomy of Breast Cancer International Consortium

MSL

Mesenchymal stem-like

PARP

poly-ADP ribose polymerase

TCGA

The Cancer Genome Atlas

TIL

tumor-infiltrating lymphocytes

TNBC

Triple-negative breast cancer

Declarations 
This study was approved by the institutional review board of Seoul St. Mary's Hospital, College of Medicine, The Catholic University of Korea (IRB no. KC17TNSI0414), and the requirement for informed consent was waived.

\section{Consent for publication}

Not applicable

\section{Availability of data and materials}

The datasets used and/or analyzed during the current study are available from the corresponding author on reasonable request.

\section{Competing interests}

The authors declare that they have no competing interests.

\section{Funding}

This work was supported by the Basic Science Research Program of the National Research Foundation funded by the Ministry of Education [grant number: 2017R1D1A1B03033486].

\section{Author Contributions}

T.-K.Y and B.-J.C performed study concept and design; T.-K.Y. and J.K. performed development of methodology and writing, review and revision of the paper; T.-K.Y., J.K., A.L. and B.-J.C. provided acquisition, analysis and interpretation of data, and statistical analysis. All authors read and approved the final paper.

\section{Acknowledgements}

We thank Yoon Jin Cha, Department of Pathology, Yonsei University College of Medicine, Seoul, Korea for evaluating tumor-infiltrating lymphocytes in our study.

\section{References}

1. Dent R, Trudeau M, Pritchard KI, Hanna WM, Kahn HK, Sawka CA, et al. Triple-negative breast cancer: clinical features and patterns of recurrence. Clin Cancer Res. 2007;13(15 Pt 1):4429-34.

2. Lee JA, Kim KI, Bae JW, Jung YH, An H, Lee ES. Triple negative breast cancer in Korea-distinct biology with different impact of prognostic factors on survival. Breast Cancer Res Treat. 2010;123(1):177-87.

3. Prat A, Adamo B, Cheang MC, Anders CK, Carey LA, Perou CM. Molecular characterization of basal-like and non-basal-like triple-negative breast cancer. Oncologist. 2013;18(2):123-33. 
4. Lehmann BD, Bauer JA, Chen X, Sanders ME, Chakravarthy AB, Shyr Y, et al. Identification of human triple-negative breast cancer subtypes and preclinical models for selection of targeted therapies. $\mathrm{J}$ Clin Invest. 2011;121(7):2750-67.

5. Liu YR, Jiang YZ, Xu XE, Yu KD, Jin X, Hu X, et al. Comprehensive transcriptome analysis identifies novel molecular subtypes and subtype-specific RNAs of triple-negative breast cancer. Breast Cancer Res. 2016;18(1):33.

6. Burstein MD, Tsimelzon A, Poage GM, Covington KR, Contreras A, Fuqua SA, et al. Comprehensive genomic analysis identifies novel subtypes and targets of triple-negative breast cancer. Clin Cancer Res. 2015;21(7):1688-98.

7. Jézéquel P, Loussouarn D, Guérin-Charbonnel C, Campion L, Vanier A, Gouraud W, et al. Geneexpression molecular subtyping of triple-negative breast cancer tumours: importance of immune response. Breast Cancer Res. 2015;17:43.

8. Masuda H, Baggerly KA, Wang Y, Zhang Y, Gonzalez-Angulo AM, Meric-Bernstam F, et al. Differential response to neoadjuvant chemotherapy among 7 triple-negative breast cancer molecular subtypes. Clin Cancer Res. 2013;19(19):5533-40.

9. Chen X, Li J, Gray WH, Lehmann BD, Bauer JA, Shyr Y, et al. TNBCtype: A Subtyping Tool for TripleNegative Breast Cancer. Cancer Inform. 2012;11:147-56.

10. Lehmann BD, Jovanović B, Chen X, Estrada MV, Johnson KN, Shyr Y, et al. Refinement of TripleNegative Breast Cancer Molecular Subtypes: Implications for Neoadjuvant Chemotherapy Selection. PLoS One. 2016;11(6):e0157368.

11. Jiang YZ, Ma D, Suo C, Shi J, Xue M, Hu X, et al. Genomic and Transcriptomic Landscape of TripleNegative Breast Cancers: Subtypes and Treatment Strategies. Cancer Cell. 2019;35(3):428-40.e425.

12. Herschkowitz $\mathrm{JI}, \mathrm{He} X$, Fan $\mathrm{C}$, Perou $\mathrm{CM}$. The functional loss of the retinoblastoma tumour suppressor is a common event in basal-like and luminal B breast carcinomas. Breast Cancer Res. 2008;10(5):R75.

13. Liu YX, Wang KR, Xing H, Zhai XJ, Wang LP, Wang W. Attempt towards a novel classification of triplenegative breast cancer using immunohistochemical markers. Oncol Lett. 2016;12(2):1240-56.

14. Stefansson OA, Jonasson JG, Olafsdottir K, Hilmarsdottir H, Olafsdottir G, Esteller M, et al. CpG island hypermethylation of BRCA1 and loss of pRb as co-occurring events in basal/triple-negative breast cancer. Epigenetics. 2011;6(5):638-49.

15. Maeda T, Nakanishi Y, Hirotani Y, Fuchinoue F, Enomoto K, Sakurai K, et al. Immunohistochemical coexpression status of cytokeratin 5/6, androgen receptor, and p53 as prognostic factors of adjuvant chemotherapy for triple negative breast cancer. Med Mol Morphol. 2016;49(1):11-21.

16. Kim S, Moon BI, Lim W, Park S, Cho MS, Sung SH. Feasibility of Classification of Triple Negative Breast Cancer by Immunohistochemical Surrogate Markers. Clin Breast Cancer. 2018;18(5):e1123-32.

17. Bareche $Y$, Venet $D$, Ignatiadis $M$, Aftimos $P$, Piccart $M$, Rothe $F$, et al. Unravelling triple-negative breast cancer molecular heterogeneity using an integrative multiomic analysis. Ann Oncol. 2018;29(4):895902. 
18. Hata T, Rajabi H, Yamamoto M, Jin C, Ahmad R, Zhang Y, et al. Targeting MUC1-C Inhibits TWIST1 Signaling in Triple-Negative Breast Cancer. Mol Cancer Ther. 2019;18(10):1744-54.

19. Wu N, Zhang J, Zhao J, Mu K, Zhang J, Jin Z, et al. Precision medicine based on tumorigenic signaling pathways for triple-negative breast cancer. Oncol Lett. 2018;16(4):4984-96.

20. Hammond ME, Hayes DF, Dowsett M, Allred DC, Hagerty KL, Badve S, et al. American Society of Clinical Oncology/College Of American Pathologists guideline recommendations for immunohistochemical testing of estrogen and progesterone receptors in breast cancer. J Clin Oncol. 2010;28(16):2784-95.

21. Bhargava R, Gerald WL, Li AR, Pan Q, Lal P, Ladanyi M, et al. EGFR gene amplification in breast cancer: correlation with epidermal growth factor receptor mRNA and protein expression and HER-2 status and absence of EGFR-activating mutations. Mod Pathol. 2005;18(8):1027-33.

22. Siroy A, Abdul-Karim FW, Miedler J, Fong N, Fu P, Gilmore H, et al. MUC1 is expressed at high frequency in early-stage basal-like triple-negative breast cancer. Hum Pathol. 2013;44(10):2159-66.

23. lacobuzio-Donahue CA, Wilentz RE, Argani P, Yeo CJ, Cameron JL, Kern SE, et al. Dpc4 protein in mucinous cystic neoplasms of the pancreas: frequent loss of expression in invasive carcinomas suggests a role in genetic progression. Am J Surg Pathol. 2000;24(11):1544-8.

24. Salgado R, Denkert C, Demaria S, Sirtaine N, Klauschen F, Pruneri G, et al. The evaluation of tumorinfiltrating lymphocytes (TILs) in breast cancer: recommendations by an International TILs Working Group 2014. Ann Oncol. 2015;26(2):259-71.

25. Moore IIDH. Classification and regression trees, by Leo Breiman, Jerome H, Friedman RA, Olshen, Stone CJ. Brooks/Cole Publishing, Monterey, 1984,358 pages. Cytometry. 1987;8(5):534-535.

26. Zhao S, Ma D, Xiao Y, Li XM, Ma JL, Zhang H, et al. Molecular Subtyping of Triple-Negative Breast Cancers by Immunohistochemistry: Molecular Basis and Clinical Relevance. Oncologist. 2020.

27. Turner NC, Reis-Filho JS. Tackling the diversity of triple-negative breast cancer. Clin Cancer Res. 2013;19(23):6380-8.

28. Lehmann BD, Pietenpol JA. Identification and use of biomarkers in treatment strategies for triplenegative breast cancer subtypes. J Pathol. 2014;232(2):142-50.

29. Collins LC, Cole KS, Marotti JD, Hu R, Schnitt SJ, Tamimi RM. Androgen receptor expression in breast cancer in relation to molecular phenotype: results from the Nurses' Health Study. Mod Pathol. 2011;24(7):924-31.

30. Park S, Koo J, Park HS, Kim JH, Choi SY, Lee JH, et al. Expression of androgen receptors in primary breast cancer. Ann Oncol. 2010;21(3):488-92.

31. Niemeier LA, Dabbs DJ, Beriwal S, Striebel JM, Bhargava R. Androgen receptor in breast cancer: expression in estrogen receptor-positive tumors and in estrogen receptor-negative tumors with apocrine differentiation. Mod Pathol. 2010;23(2):205-12.

32. McGhan LJ, McCullough AE, Protheroe CA, Dueck AC, Lee JJ, Nunez-Nateras R, et al. Androgen receptor-positive triple negative breast cancer: a unique breast cancer subtype. Ann Surg Oncol. 2014;21(2):361-7. 
33. Gucalp A, Tolaney S, Isakoff SJ, Ingle JN, Liu MC, Carey LA, et al. Phase II Trial of Bicalutamide in Patients with Androgen Receptor-Positive, Estrogen Receptor-Negative Metastatic Breast Cancer. Clin Cancer Res. 2013;19(19):5505-12.

34. Traina TA, Miller K, Yardley DA, Eakle J, Schwartzberg LS, O'Shaughnessy J, et al. Enzalutamide for the Treatment of Androgen Receptor-Expressing Triple-Negative Breast Cancer. J Clin Oncol. 2018;36(9):884-90.

35. Gonzalez-Ericsson PI, Stovgaard ES, Sua LF, Reisenbichler E, Kos Z, Carter JM, et al. The path to a better biomarker: application of a risk management framework for the implementation of PD-L1 and TILs as immuno-oncology biomarkers in breast cancer clinical trials and daily practice. J Pathol. 2020;250(5):667-84.

36. Schmid P, Adams S, Rugo HS, Schneeweiss A, Barrios CH, Iwata H, et al. Atezolizumab and NabPaclitaxel in Advanced Triple-Negative Breast Cancer. N Engl J Med. 2018;379(22):2108-21.

37. Emens LA, Loi S, Rugo HS, Schneeweiss A, Diéras V, Iwata H, et al. IMpassion130: efficacy in immune biomarker subgroups from the global, randomized, double-blind, placebo-controlled, phase III study of atezolizumab + nab-paclitaxel in patients with treatment-naïve, locally advanced or metastatic triplenegative breast cancer. In: San Antonio Breast Cancer Symposium: 2018; 2018.

38. Loi S, Winer E, Lipatov O, Im S-A, Goncalves A, Cortes J, et al. Abstract PD5-03: Relationship between tumor-infiltrating lymphocytes (TILs) and outcomes in the KEYNOTE-119 study of pembrolizumab vs chemotherapy for previously treated metastatic triple-negative breast cancer (mTNBC). Cancer Res. 2020;80(4 Supplement):PD5-03-PD05-03.

39. Loi S, Adams S, Schmid P, Cortés J, Cescon D, Winer E, et al. Relationship between tumor infiltrating lymphocyte (TIL) levels and response to pembrolizumab (pembro) in metastatic triple-negative breast cancer (mTNBC): Results from KEYNOTE-086. Ann Oncol. 2017;28:v608.

40. Zou Y, Zou X, Zheng S, Tang H, Zhang L, Liu P, et al. Efficacy and predictive factors of immune checkpoint inhibitors in metastatic breast cancer: a systematic review and meta-analysis. Ther Adv Med Oncol. 2020;12:1758835920940928.

41. Carey LA, Rugo HS, Marcom PK, Mayer EL, Esteva FJ, Ma CX, et al. TBCRC 001: randomized phase II study of cetuximab in combination with carboplatin in stage IV triple-negative breast cancer. J Clin Oncol. 2012;30(21):2615-23.

42. Bell R, Brown J, Parmar M, Toi M, Suter T, Steger GG, et al. Final efficacy and updated safety results of the randomized phase III BEATRICE trial evaluating adjuvant bevacizumab-containing therapy in triplenegative early breast cancer. Ann Oncol. 2017;28(4):754-60.

43. Hara E, Smith R, Parry D, Tahara H, Stone S, Peters G. Regulation of p16CDKN2 expression and its implications for cell immortalization and senescence. Mol Cell Biol. 1996;16(3):859-67.

44. Romagosa C, Simonetti S, López-Vicente L, Mazo A, Lleonart ME, Castellvi J, et al. p16(Ink4a) overexpression in cancer: a tumor suppressor gene associated with senescence and high-grade tumors. Oncogene. 2011;30(18):2087-97. 
45. Bogina GS, Lunardi G, Marcolini L, Brunelli M, Bortesi L, Marconi M, et al. P16 but not retinoblastoma expression is related to clinical outcome in no-special-type triple-negative breast carcinomas. Mod Pathol. 2014;27(2):204-13.

46. Garsed DW, Alsop K, Fereday S, Emmanuel C, Kennedy CJ, Etemadmoghadam D, et al. Homologous Recombination DNA Repair Pathway Disruption and Retinoblastoma Protein Loss Are Associated with Exceptional Survival in High-Grade Serous Ovarian Cancer. Clin Cancer Res. 2018;24(3):569-80.

47. Galot R, Le Tourneau C, Guigay J, Licitra L, Tinhofer I, Kong A, et al. Personalized biomarker-based treatment strategy for patients with squamous cell carcinoma of the head and neck: EORTC position and approach. Ann Oncol. 2018;29(12):2313-27.

48. Robson M, Im SA, Senkus E, Xu B, Domchek SM, Masuda N, et al. Olaparib for Metastatic Breast Cancer in Patients with a Germline BRCA Mutation. N Engl J Med. 2017;377(6):523-33.

49. Litton JK, Rugo HS, Ettl J, Hurvitz SA, Gonçalves A, Lee KH, et al. Talazoparib in Patients with Advanced Breast Cancer and a Germline BRCA Mutation. N Engl J Med. 2018;379(8):753-63.

50. Karaayvaz M, Cristea S, Gillespie SM, Patel AP, Mylvaganam R, Luo CC, et al. Unravelling subclonal heterogeneity and aggressive disease states in TNBC through single-cell RNA-sEq. Nat Commun. 2018;9(1):3588.

51. James G, Witten D, Hastie T, Tibshirani R, SpringerLink. An Introduction to Statistical Learning: with Applications in R. New York: Imprint: Springer;: Springer; 2013.

\section{Figures}


LAR
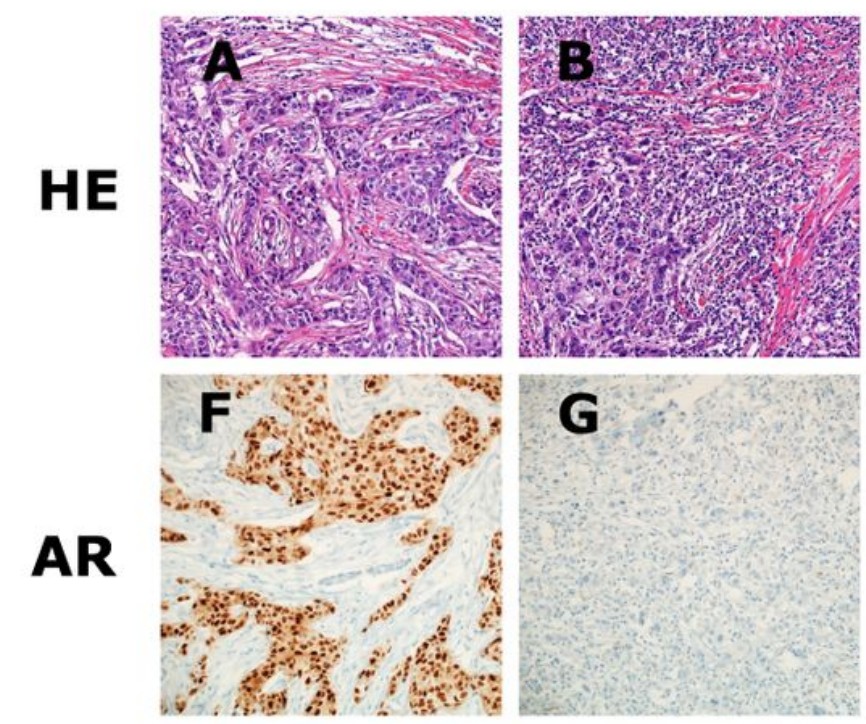

p16

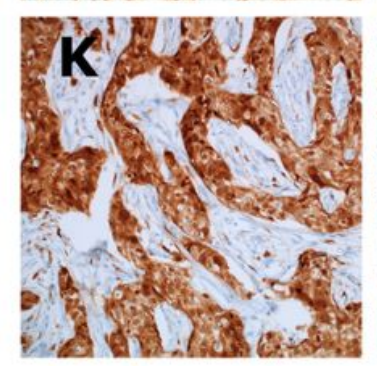

IM
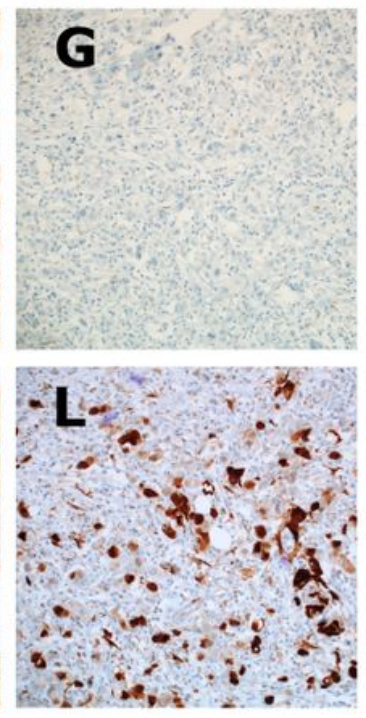

M
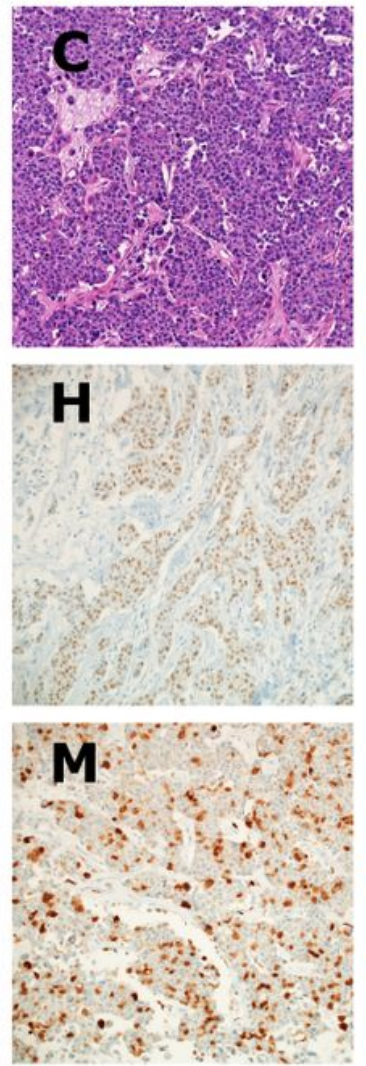

BL1

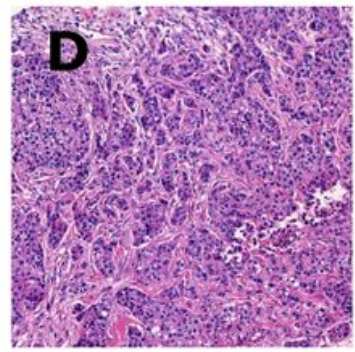

I

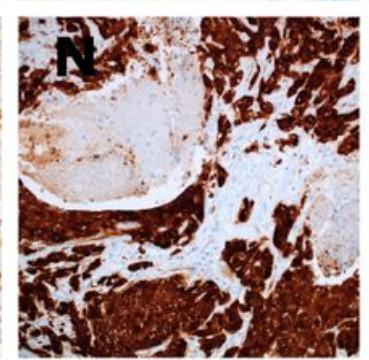

UNC
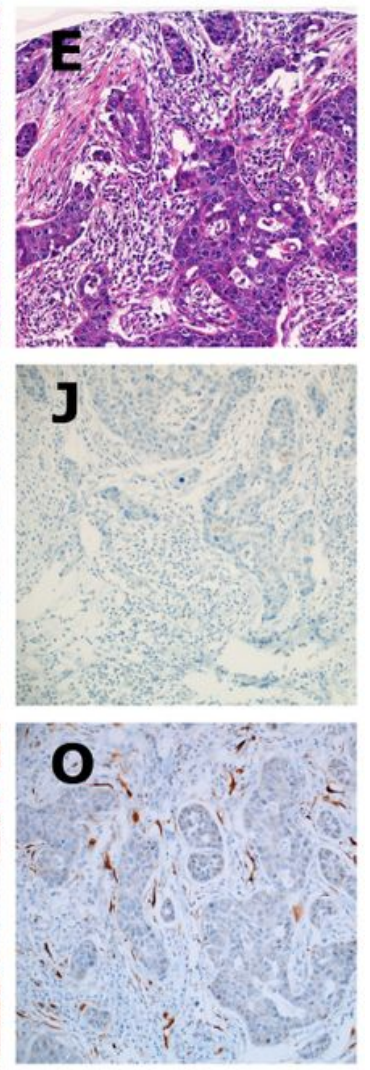

\section{Figure 1}

Representative images of TNBC surrogate classification. Hematoxylin and eosin (H\&E) stain (A-E), tumorinfiltrating lymphocytes (TIL); A:20, B:90, C:10, D:20, E: 60. Androgen receptor immunohistochemical stain (F-J); Allred score F:8, G:0, H:1, I:0, J:1. p16 immunohistochemical stain (K-0); K, N: diffuse and strong, L, M: weak and mosaic, 0 : negative. 


\section{TNBC}

AR?

LAR subtype

AR Allred score 0-7

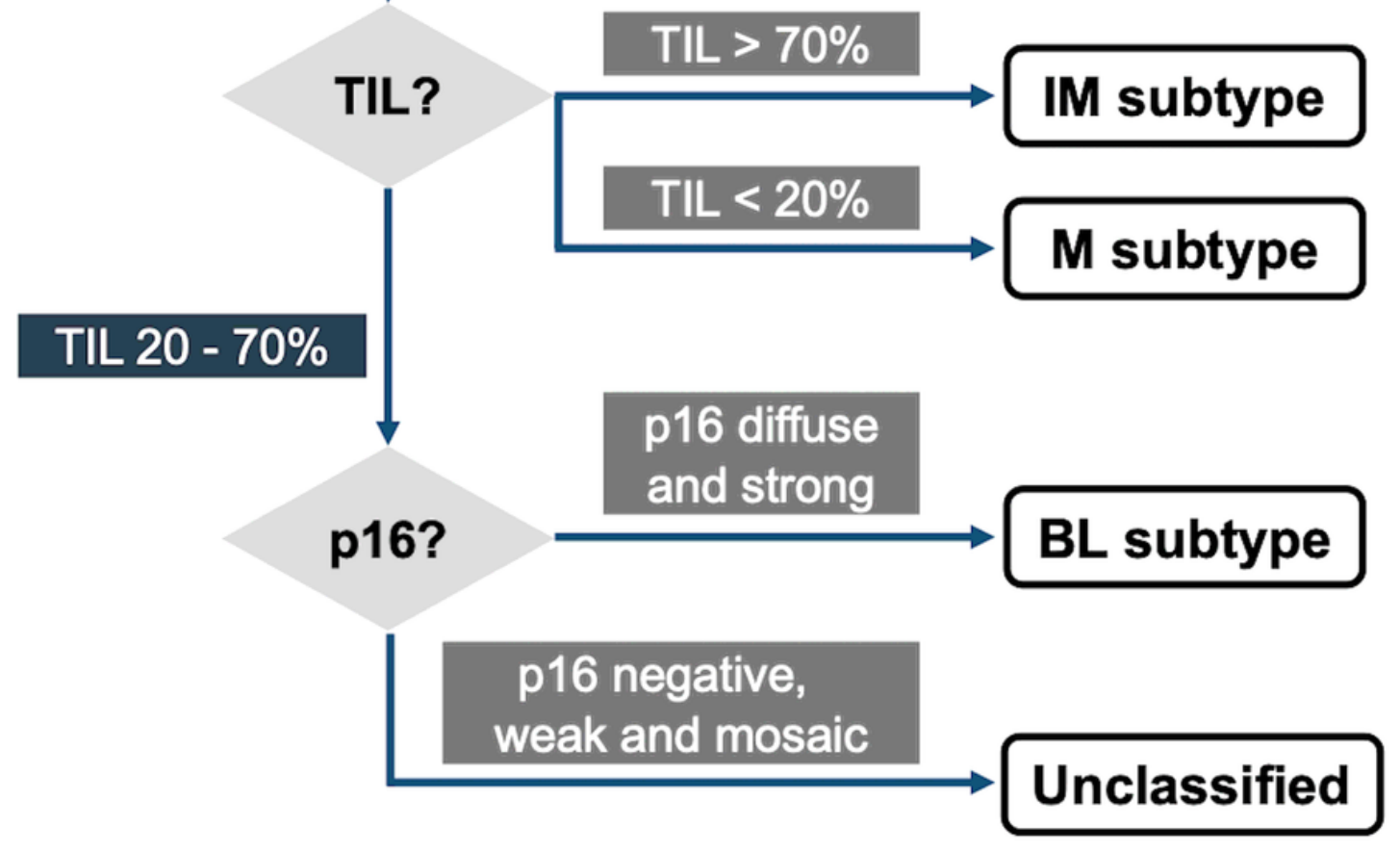

Figure 2

TNBC surrogate subtype classification method 
A

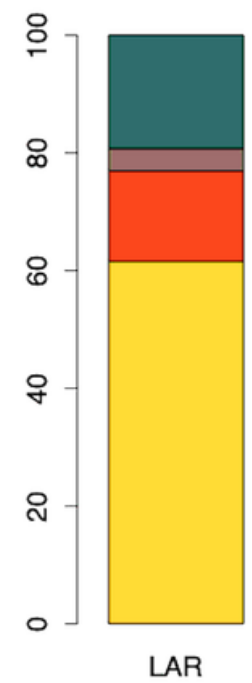

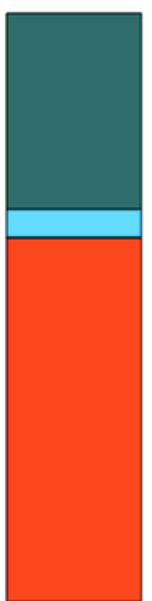

IM

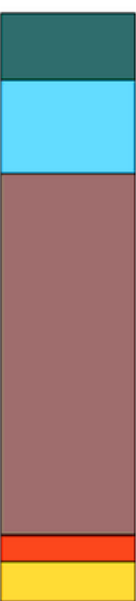

$\mathrm{M}$

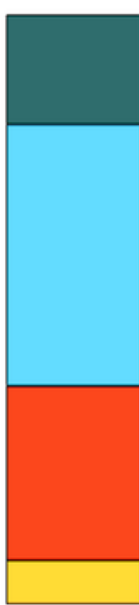

$\mathrm{BL}$

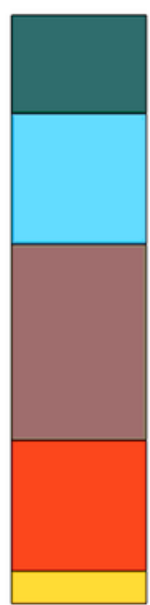

UNC
B

\begin{tabular}{|c|c|c|c|c|c|}
\hline $\begin{array}{c}\text { SUR } \\
\text { RNA }\end{array}$ & LAR & IM & M & BL & UNC \\
\hline LAR & 16 & 0 & 3 & 2 & 1 \\
\hline IM & 4 & 13 & 2 & 8 & 4 \\
\hline$M$ & 1 & 0 & 27 & 0 & 6 \\
\hline BL & 0 & 1 & 7 & 12 & 4 \\
\hline UNC & 5 & 7 & 5 & 5 & 3 \\
\hline
\end{tabular}

RNA: $\because \mathrm{LAR}=\mathrm{IM} \backsim \mathrm{M} \backsim \mathrm{BL} \backsim \mathrm{UNC}$

\section{Figure 3}

Concurrence between TNBC surrogate subtype classification and gene expression-based classification (Vanderbilt 4 subtypes). (A) Bar chart showing concurrence between TNBC surrogate subtypes and Vanderbilt 4 subtypes. (B) Confusion matrix for concurrence between TNBC surrogate subtypes and Vanderbilt 4 subtypes. SUR, TNBC surrogate subtype classification; UNC, unclassified 


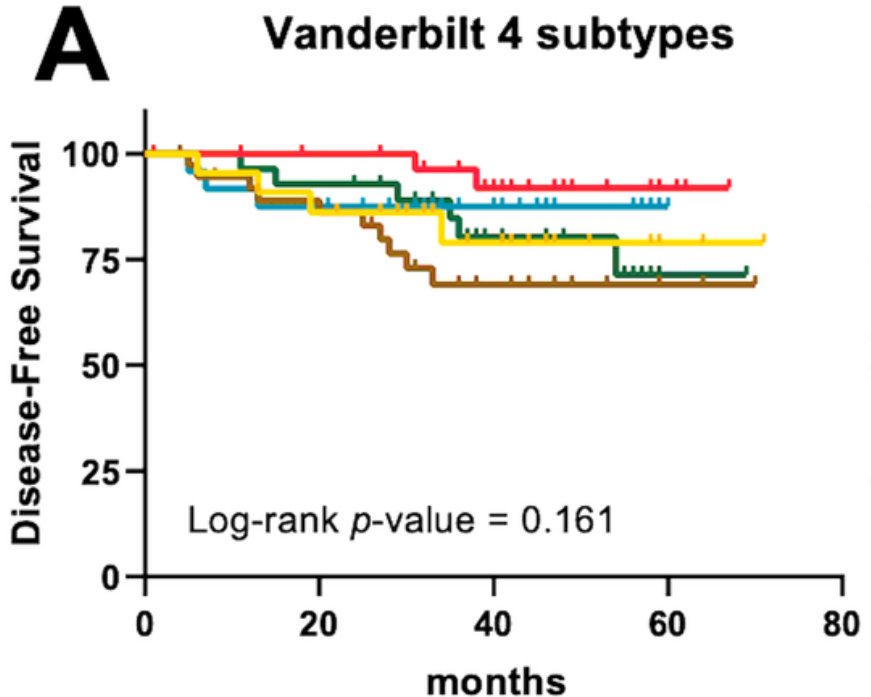

B TNBC Surrogate Classification

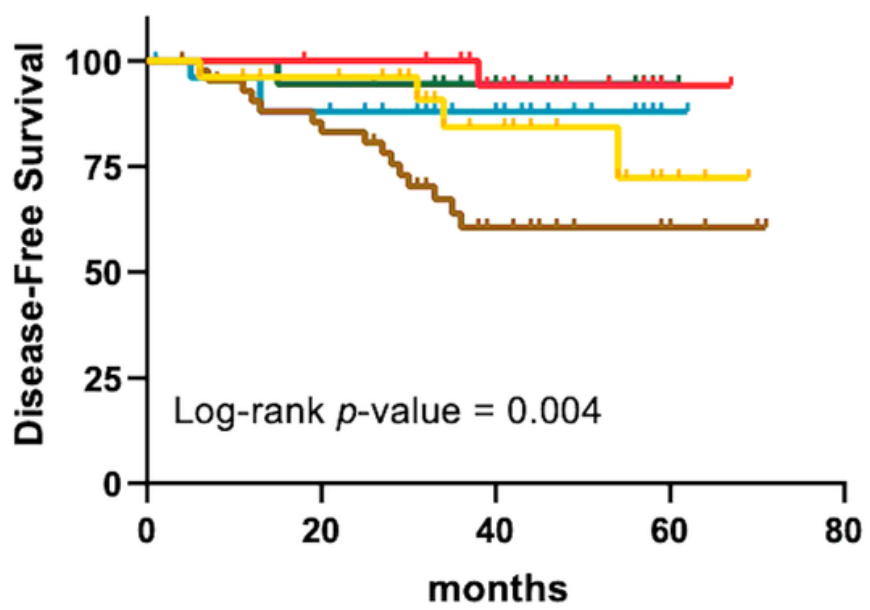

Vanderbilt 4 subtypes

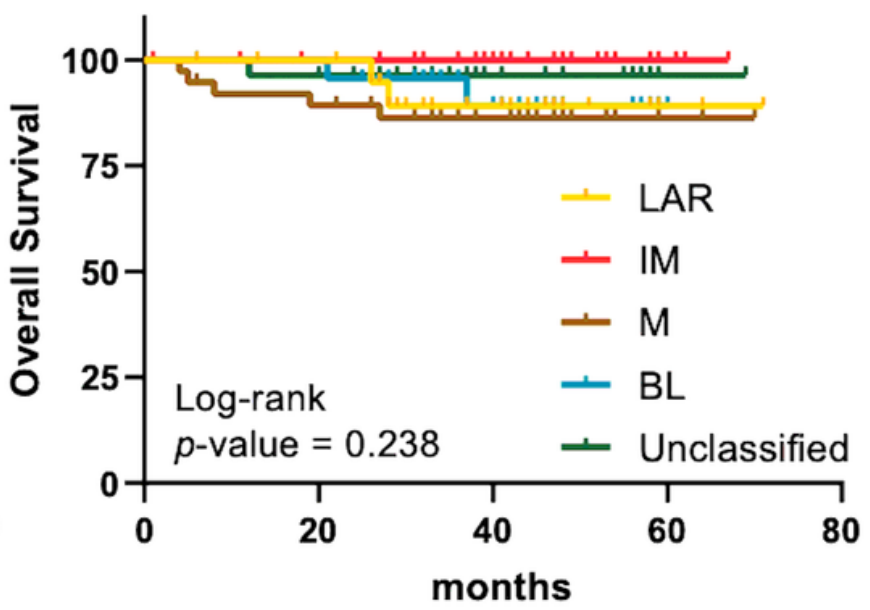

TNBC Surrogate Classification

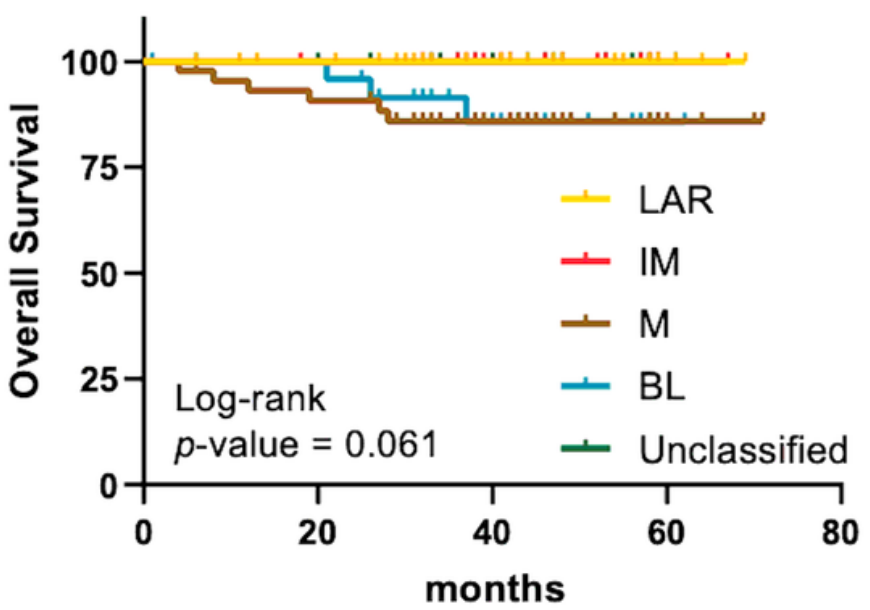

Figure 4

Kaplan-Meier curves of disease-free survival and overall survival according to Vanderbilt 4 subtypes and surrogate subtypes

\section{Supplementary Files}

This is a list of supplementary files associated with this preprint. Click to download.

- Additionalfile1.pdf 